\title{
Efeitos Cardiovasculares de Duas Doses de Dexmedetomidina. Estudo Experimental em Cães *
}

\section{Cardiovascular Effects of Two Dexmedetomidine Doses. Experimental Study in Dogs}

\author{
Nivaldo Ribeiro Villela ${ }^{1}$; Paulo do Nascimento Júnior, TSA ${ }^{2}$; Lídia Raquel de Carvalho ${ }^{3}$
}

\section{RESUMO}

Villela NB, Nascimento Jr P, Carvalho LR - Efeitos Cardiovasculares de Duas Doses de Dexmedetomidina. Estudo Experimental em Cães

\begin{abstract}
JUSTIFICATIVA E OBJETIVOS: A dexmedetomidina é um novo agonista $\alpha_{2}$-adrenérgico, havendo, atualmente, crescente interesse no seu uso em Anestesiologia, por reduzir o consumo de anestésicos e promover estabilidade hemodinâmica. O objetivo desta pesquisa foi estudar os efeitos cardiovasculares da dexmedetomidina no cão anestesiado, empregando-se duas doses distintas e semelhantes àquelas utilizadas em Anestesiologia.
\end{abstract}

MÉTODO: 36 cães adultos anestesiados com propofol, fentanil e isoflurano foram divididos em três grupos: G1, injeção de 20 $\mathrm{ml}$ de solução de cloreto de sódio a 0,9\%, em 10 minutos, seguida de injeção de $20 \mathrm{ml}$ da mesma solução, em 1 hora; G2, contendo dexmedetomidina $\left(1 \mu \mathrm{g} \cdot \mathrm{kg}^{-1}\right)$, em 10 minutos, seguida de injeção de $20 \mathrm{ml}$ da mesma solução, em 1 hora e G3, injeção de $20 \mathrm{ml}$ de solução de cloreto de sódio a $0,9 \%$ de injeção de $20 \mathrm{ml}$ da mesma solução, em 1 hora. Estudaram-se os atributos cardiovasculares em quatro momentos: $M_{1}$, controle; $M_{2}$, após a injeção inicial de $20 \mathrm{ml}$ da solução em estudo, em 10 minutos, coincidindo com o início da injeção da mesma solução, em 1 hora; $M_{3}, 60$ minutos após $M_{2}$ e $M_{4}, 60$ minutos após $M_{3}$.

RESULTADOS: A freqüência cardíaca (FC) diminuiu no $G 2$, no $M_{2}$, retornando aos valores basais no $M_{3}$, enquanto no $G 3$ diminuiu no $\mathrm{M}_{2}$, mantendo-se baixa durante todo o experimento. No G1 houve aumento progressivo da FC. Em nenhum grupo houve alteração da pressão arterial. A resistência vascular sistêmica (RVS) manteve-se estável no G2 e G3, enquanto no $G 1$ apresentou redução em $M_{2}$, mantendo-se baixa ao longo do experimento. O índice cardíaco (IC) não apresentou alterações significativas no G2 e G3, mas aumentou progressivamente no G1.

\footnotetext{
* Recebido do (Received from) Departamento de Anestesiologia da Faculdade de Medicina de Botucatu (FMB - UNESP), Botucatu, SP

1. Mestre em Anestesiologia pela FMB - UNESP. Pós-Graduando, Doutorado, do Departamento de Anestesiologia da FMB - UNESP

2. Professor Assistente Doutor do Departamento de Anestesiologia da FMB - UNESP

3. Professora Assistente Doutora do Departamento de Bioestatística do Instituto de Biociências de Botucatu, UNESP
}

Apresentado (Submitted) em 14 de janeiro de 2003

Aceito (Accepted) para publicação em 21 de março de 2003

Endereço para correspondência (Correspondence to) Dr. Nivaldo Ribeiro Villela

Av. Henrique Dodsworth, 133/1407 - Lagoa

22061-030 Rio de Janeiro, RJ

E-mail: nivaldovillela@terra.com.br

(c) Sociedade Brasileira de Anestesiologia, 2003 injeção de $20 \mathrm{ml}$ de solução de cloreto de sódio a $0,9 \%$ contendo dexmedetomidina $\left(2 \mu \mathrm{g} \cdot \mathrm{kg}^{-1}\right)$ em 10 minutos, seguida

CONCLUSÕES: Conclui-se que no cão, nas condições experimentais empregadas, a dexmedetomidina diminui a FC de forma dose-dependente, inibe a redução da RVS produzida pelo isoflurano e impede a ocorrência de resposta hiperdinâmica durante o experimento.

Unitermos: ANIMAL: cão; DROGAS, $\alpha_{2}$-agonistas: dexmedetomidina

\section{SUMMARY}

Villela NB, Nascimento Jr P, Carvalho LR - Cardiovascular Effects of Two Dexmedetomidine Doses. Experimental Study in Dogs

BACKGROUND AND OBJECTIVES: There has been a growing interest in the anesthetic use of dexmedetomidine, a new $\alpha_{2}$-adrenergic agonist, due to decreased anesthetics consumption and better cardiovascular stability that it promoter. This study aimed at investigating cardiovascular effects of two different dexmedetomidine doses in anesthetized dogs.

METHODS: The study involved 36 adult dogs anesthetized with propofol, fentanyl and isoflurane distributed in three groups which received: $G 1,20 \mathrm{ml}$ saline injection in 10 minutes, followed by $20 \mathrm{ml}$ of the same solution infused in one hour; G2, 20 $\mathrm{ml}$ dexmedetomidine-containing saline $\left(1 \mu \mathrm{g} . \mathrm{kg}^{-1}\right)$ in $10 \mathrm{~min}$ utes, followed by $20 \mathrm{ml}$ of the same solution infused one hour; and $\mathrm{G} 3$, of dexmedetomidine-containing saline $\left(2 \mu \mathrm{g} \cdot \mathrm{kg}^{-1}\right)$ in 10 minutes, followed by $20 \mathrm{ml}$ of the same solution infused in one hour. Cardiovascular attributes were evaluated in four moments: $M_{1}$, control, $M_{2}$, after initial $20 \mathrm{ml}$ injection of the studied solution, coincident with the beginning of the same solution injection in one hour; $M_{3}, 60$ minutes after $M_{2}$ and $M_{4}, 60$ minutes after $\mathrm{M}_{3}$.

RESULTS: $G 2$ heart hate (HR) was decreased at $M_{2}$, returning to baseline values at $M_{3}$, while in $G 3$ it was decreased at $M_{2}$ but was kept so throughout the experiment. There has been progressive HR increase in G1. There were no significant changes in mean blood pressure in all group. Systemic vascular resistance (SVR) was maintained stable in G2 and G3, but was decreased in $\mathrm{G} 1$ at $\mathrm{M}_{3}$. Cardiac index (Cl) was not significantly changed in $\mathrm{G} 2$ and $\mathrm{G} 3$, but has progressively increased in $\mathrm{G} 1$.

CONCLUSIONS: In dogs under these experimental conditions, dexmedetomidine has induced a dose-dependent HR decrease, has inhibited isoflurane-induced SVR decrease and has prevented hyperdynamic responses throughout the experiment.

Key Words: ANIMAL: dog; DRUGS, $\alpha_{2}$-agonist: dexmedetomidine

\section{INTRODUÇÃO}

Ativação do sistema nervoso simpático (SNS), decorArente de vários procedimentos anestésicos e cirúrgicos, pode provocar, em pacientes com doença coronariana, instabilidade hemodinâmica e alterações metabólicas ${ }^{1}$, aumentando a incidência de isquemia ou infarto do miocárdio 
e de graves disritmias ${ }^{2}$. Com intenção de minimizar essa resposta cardiovascular hiperdinâmica, há um crescente interesse no uso dos agonistas $\alpha_{2}$-adrenérgicos durante o período per-operatório como drogas coadjuvantes da anestesia $^{3}$.

Adexmedetomidina, o enantiômero dextrógiro da medetomidina, é um agonista $\alpha_{2}$-adrenérgico superseletivo que apresenta relação de seletividade entre os receptores $\alpha_{2}: \alpha_{1}$ de $1600: 1^{4}$, tendo demonstrado, em estudos experimentais, importante ação sedativa e analgésica ${ }^{5}$, reduzindo o consumo de anestésicos e opióides durante anestesia ${ }^{6}$.

Em voluntários sadios, a dexmedetomidina decresce em até $90 \%$ a concentração plasmática das catecolaminas ${ }^{7,8}$, promove sedação, hipnose e analgesia ${ }^{9}$. Em pacientes cirúrgicos, a dexmedetomidina reduz a necessidade de anestési$\cos ^{10-12}$ e opióides ${ }^{13,14}$, mantém maior estabilidade hemodinâmica ${ }^{10,15}$ e diminui o consumo de analgésicos e sedativos no pós-operatório ${ }^{16}$.

Os efeitos cardiovasculares da dexmedetomidina são caracterizados principalmente por redução da pressão arterial média (PAM) e da freqüência cardíaca (FC), cuja intensidade das alterações depende da dose utilizada, da forma de administração da droga, da espécie e do tônus simpático do animal que recebe o medicamento ${ }^{17}$. Bradicardia e hipotensão arterial são efeitos adversos observados com o emprego da dexmedetomidina ${ }^{18}$, o que tem sido motivo de preocupação entre os anestesiologistas ${ }^{19}$.

Na literatura, há escassez de trabalhos experimentais em que se utilize a dexmedetomidina em condições semelhantes àquelas praticadas nas salas de operações. Assim, o objetivo desta pesquisa foi estudar os efeitos hemodinâmicos cardiovasculares da dexmedetomidina em modelo experimental no cão anestesiado, empregando-se duas doses distintas e semelhantes àquelas utilizadas na prática clínica em Anestesiologia.

\section{MÉTODO}

Esta pesquisa foi aprovada pela Comissão de Ética na Experimentação Animal da Faculdade de Medicina de Botucatu, UNESP. Foram utilizados 36 cães adultos de ambos os sexos, sem raça definida, com peso entre 18 e $30 \mathrm{~kg}$.

\section{Preparo Anestésico-Cirúrgico}

Após período de jejum alimentar de 12 horas, com livre acesso à água, os animais foram anestesiados com propofol (6 $\left.\mathrm{mg} \cdot \mathrm{kg}^{-1}\right)$ e fentanil $\left(5 \mu \mathrm{g} \cdot \mathrm{kg}^{-1}\right)$. Após a intubação traqueal, os pulmões foram ventilados mecanicamente com oxigênio $\left(0,8\right.$ L. $\left.\mathrm{min}^{-1}\right)$ e ar comprimido $\left(1,2 \mathrm{~L}\right.$. $\left.\mathrm{min}^{-1}\right)$, com volume corrente de $20 \mathrm{ml} . \mathrm{kg}^{-1}$ e freqüência respiratória de 12 a 16 movimentos por minuto, com objetivo de manter a pressão expiratória final de $\mathrm{CO}_{2}\left(\mathrm{P}_{\mathrm{ET}} \mathrm{CO}_{2}\right)$ em $35 \mathrm{a} 45 \mathrm{mmHg}$, e iniciou-se administração de isoflurano em concentração expirada de 1,7 CAM. Realizou-se dissecção e cateterismo da veia femoral direita para início da hidratação com solução de Ringer com lactato $\left(18 \mathrm{ml} \cdot \mathrm{kg}^{-1} \cdot \mathrm{h}^{-1}\right)$ e administrou-se o brometo de rocurônio $\left(0,6 \mathrm{mg} \cdot \mathrm{kg}^{-1}\right.$ e injeção contínua de $\left.10 \mu \mathrm{g} \cdot \mathrm{kg}^{-1} \cdot \mathrm{min}^{-1}\right)$. Aseguir foram dissecadas e cateterizadas a artéria femoral esquerda, para medida contínua da PAM, a veia femoral esquerda, para coleta de sangue para dosagem do hematócrito $(\mathrm{Ht})$, e a veia jugular externa direita, onde foi posicionado o introdutor e passado o cateter de Swan-Ganz 7F até a artéria pulmonar para medida do débito cardíaco por termodiluição, da pressão média do átrio direito (PAD), da pressão média da artéria pulmonar (PAP) e da pressão da artéria pulmonar ocluída (PAPO). A manutenção da temperatura dos animais foi feita através de insuflação de ar aquecido na superfície ventral $\left(38\right.$ a $\left.42^{\circ} \mathrm{C}\right)$ e aquecimento das soluções injetadas. A temperatura foi monitorizada por meio de sensor localizado no esôfago.

Após o término do preparo cirúrgico, as feridas foram infiltradas com ropivacaína a $0,2 \%$, a concentração expirada do isoflurano foi reduzida para 0,6 CAM e o volume de injeção da solução de Ringer com lactato diminuído para $6 \mathrm{ml} \cdot \mathrm{kg}^{-1} \cdot \mathrm{h}^{-1}$. Iniciou-se período de estabilização hemodinâmica de $30 \mathrm{mi}$ nutos e, em seguida, foi realizado o primeiro momento de estudo (controle).

\section{Grupos Estudados}

Após o momento controle, os animais foram distribuídos de forma aleatória e encoberta em três grupos, com 12 cães em cada grupo:

G1 ( $n=12)$ : injeção de $20 \mathrm{ml}$ de solução de cloreto de sódio a $0,9 \%$, em 10 minutos, seguida de injeção de $20 \mathrm{ml}$ da mesma solução em 1 hora.

$\mathrm{G} 2(\mathrm{n}=12)$ : injeção de $20 \mathrm{ml}$ de solução de cloreto de sódio a $0,9 \%$ contendo dexmedetomidina $1 \mu \mathrm{g} \cdot \mathrm{kg}^{-1}$, em $10 \mathrm{mi}-$ nutos, seguida de injeção de $20 \mathrm{ml}$ da mesma solução, com a mesma dose de dexmedetomidina $\left(1 \mu \mathrm{g} \cdot \mathrm{kg}^{-1}\right)$, em 1 hora.

G3 ( $n=12)$ : injeção de $20 \mathrm{ml}$ de solução de cloreto de sódio a $0,9 \%$ contendo dexmedetomidina $2 \mu \mathrm{g} \cdot \mathrm{kg}^{-1}$, em $10 \mathrm{mi}-$ nutos, seguida de injeção de $20 \mathrm{ml}$ da mesma solução, com a mesma dose de dexmedetomidina $\left(2 \mu \mathrm{g} \cdot \mathrm{kg}^{-1}\right)$, em 1 hora.

\section{Atributos Estudados}

Foram estudados os seguintes atributos: para controle da homogeneidade do estudo - comprimento, peso, área de superfície corporal (ASC), sexo, hematócrito $(\mathrm{Ht})$, $\mathrm{P}_{\mathrm{ET}} \mathrm{CO}_{2}$, saturação de pulso de oxigênio $\left(\mathrm{SpO}_{2}\right)$ e temperatura esofágica $(T)$; atributos para atender as finalidades do experimento - FC, PAM, PAP, PAD, PAPO, índice cardíaco (IC), índice sistólico (IS), índice da resistência vascular sistêmica (IRVS), índice da resistência vascular pulmonar (IRVP), índice de trabalho sistólico do ventrículo esquerdo (ITSVE) e índice de trabalho sistólico do ventrículo direito (ITSVD). 


\section{Momentos Estudados}

Os atributos foram estudados em 4 momentos:

$\mathrm{M}_{1}$ (controle): imediatamente após o período de estabilização anestésica.

$\mathrm{M}_{2}$ : após a injeção inicial, em 10 minutos, de $20 \mathrm{ml}$ da solução em estudo, coincidindo com o início da injeção contínua da solução em estudo, em 1 hora.

$M_{3}$ : 60 minutos após o término de $M_{2}$, coincidindo com o término da injeção, durante 1 hora, da solução em estudo.

$\mathrm{M}_{4}$ : 60 minutos após o término de $\mathrm{M}_{3}$.

\section{Análise Estatística}

Para as variáveis que apresentaram distribuição normal e homogeneidade de variâncias, foi utilizada a Análise de Perfil, seguida do método de Tukey para comparações múltiplas. Para as que não apresentaram distribuição normal ou homogeneidade de variâncias, foi utilizado o teste de Friedman para comparações dos momentos e o teste de Kruskal-Wallis para comparação dos grupos, seguidos do teste para comparações múltiplas. Os dados demográficos foram verificados pelo método de Análise de Variância. Com relação ao sexo, empregou-se o teste Exato de
Fisher para análise de freqüência. O nível de significância utilizado foi de $5 \%$.

\section{RESULTADOS}

Os grupos mostraram-se homogêneos em relação ao peso, comprimento, área de superfície corporal (ASC) e sexo (Tabela I)

Tabela I - Peso, Comprimento, Área de Superfície Corporal (ASC) e Distribuição de Machos e Fêmeas em Cada um dos Grupos Estudados

\begin{tabular}{lcccc}
\hline Grupos & $\begin{array}{c}\mathrm{G} 1 \\
(\mathrm{n}=12)\end{array}$ & $\begin{array}{c}\mathrm{G} 2 \\
(\mathrm{n}=12)\end{array}$ & $\begin{array}{c}\mathrm{G} 3 \\
(\mathrm{n}=12)\end{array}$ & Estatística \\
\hline Peso $(\mathrm{kg})^{*}$ & $24,3 \pm 4,2$ & $21,9 \pm 3,9$ & $23,9 \pm 3,7$ & $\mathrm{p}>0,05$ \\
Comprimento $(\mathrm{cm})^{*}$ & $114,7 \pm 6,9$ & $107,3 \pm 8,0$ & $114,8 \pm 10,1$ & $\mathrm{p}>0,05$ \\
ASC $\left(\mathrm{m}^{2}\right)^{*}$ & $0,90 \pm 0,09$ & $0,85 \pm 0,09$ & $0,86 \pm 0,11$ & $\mathrm{p}>0,05$ \\
Sexo & & & & $\mathrm{p}>0,05$ \\
$\quad$ Machos & 9 & 10 & 12 & \\
\multicolumn{1}{c}{ Fêmeas } & 3 & 2 & 0 & \\
\hline
\end{tabular}

* Valores expressos pela Média \pm DP

Os resultados das variáveis hemodinâmicas, $\mathrm{Ht}, \mathrm{P}_{\mathrm{ET}} \mathrm{CO}_{2} \mathrm{e}$ $\mathrm{SpO}_{2}$ estão registrados nas tabelas II, III, IV e figuras 1, 2, 3 e 4 .

Tabela II - Valores da Freqüência Cardíaca (FC), Pressão Arterial Média (PAM), Pressão Média da Artéria Pulmonar (PAP), Pressão Média do Átrio Direito (PAD), Pressão da Artéria Pulmonar Ocluída (PAPO) e do Índice Cardíaco (IC) nos Momentos e Grupos Estudados

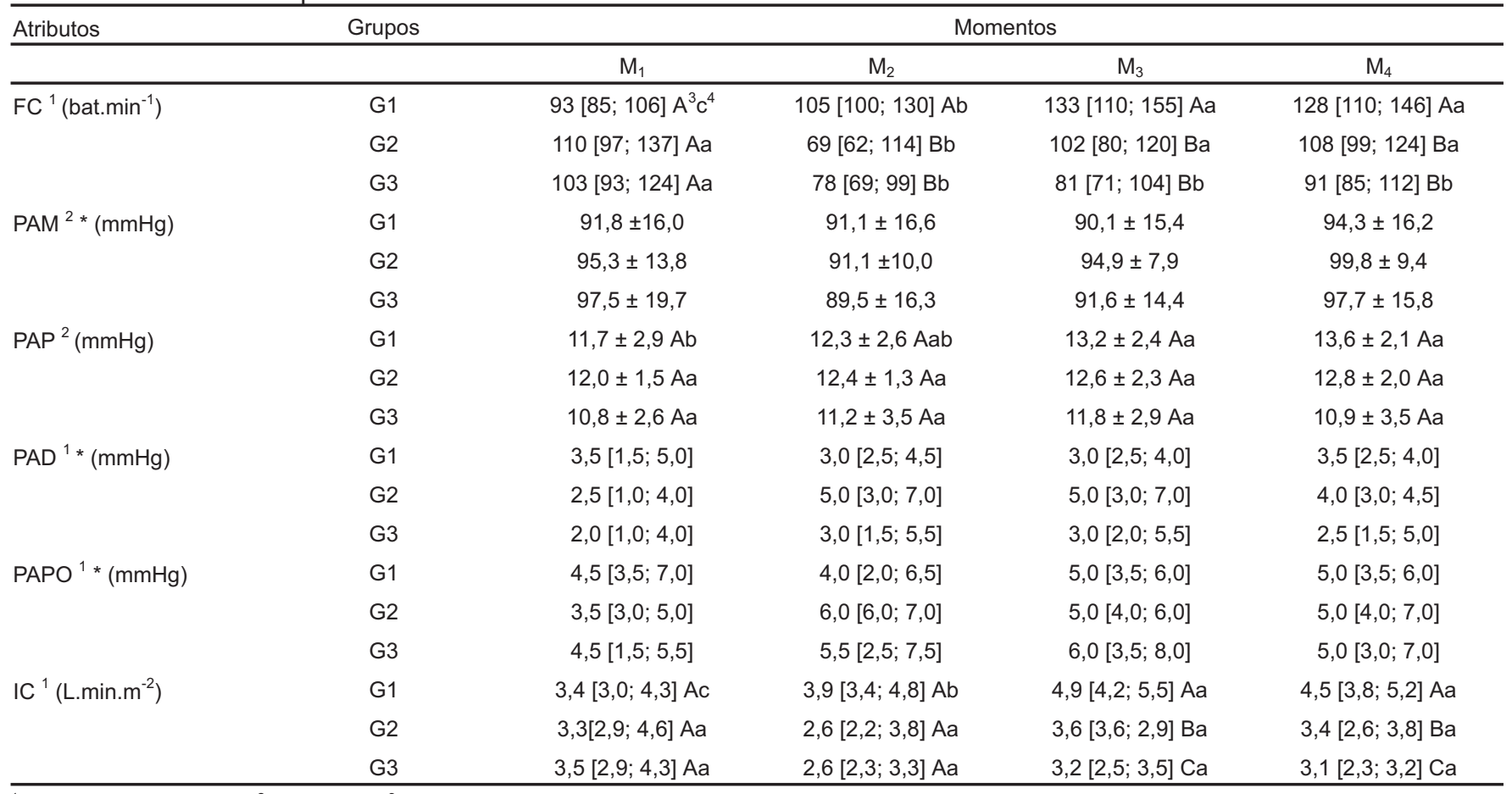

${ }^{1}$ Mediana, $1^{\circ}$ e $3^{\circ}$ quartis; ${ }^{2}$ Média $\pm D P ;{ }^{3}$ Grupos dentro de cada momento, seguidos de mesma letra maiúscula, não diferem estatisticamente ( $>0,05$ );

${ }^{4}$ Momentos dentro de cada grupo, seguidos de mesma letra minúscula, não diferem estatisticamente $(p>0,05)$; ${ }^{*} p>0,05$ (entre grupos, dentro de cada momento e entre momentos, dentro de cada grupo); Obs: $\mathrm{A}>\mathrm{B}>\mathrm{C} ; \mathrm{a}>\mathrm{b}>\mathrm{c}$ 
Tabela III - Valores do Índice Sistólico (IS), Índice da Resistência Vascular Sistêmica (IRVS), Índice da Resistência Vascular Pulmonar (IRVP), Índice de Trabalho Sistólico do Ventrículo Esquerdo (ITSVE), Índice de Trabalho Sistólico do Ventrículo Direito (ITSVD) nos Momentos e Grupos Estudados

\begin{tabular}{|c|c|c|c|c|c|}
\hline \multirow[t]{2}{*}{ Atributos } & \multirow[t]{2}{*}{ Grupos } & \multicolumn{4}{|c|}{ Momentos } \\
\hline & & $\mathrm{M}_{1}$ & $\mathrm{M}_{2}$ & $\mathrm{M}_{3}$ & $\mathrm{M}_{4}$ \\
\hline \multirow[t]{3}{*}{$\mathrm{IS}^{2 *}\left(\mathrm{ml} \cdot\right.$ bat. $\left.\mathrm{min}^{-1}\right)$} & G1 & $33,0 \pm 10,9$ & $36,6 \pm 8,9$ & $37,7 \pm 7,6$ & $35,7 \pm 5,8$ \\
\hline & G2 & $34,0 \pm 11,9$ & $37,9 \pm 9,2$ & $36,8 \pm 11,5$ & $31,9 \pm 9,9$ \\
\hline & G3 & $34,4 \pm 8,0$ & $36,4 \pm 10,0$ & $35,8 \pm 9,9$ & $29,5 \pm 7,1$ \\
\hline & G2 & $2127[1770 ; 2536] \mathrm{Aa}$ & 2490 [1709; 3267] Aa & $2069[1721 ; 2478] \mathrm{Aa}$ & $2284[1990 ; 2887] \mathrm{Aa}$ \\
\hline & G3 & 2278 [1827; 2457] Aa & 2414 [2034; 2923] Aa & $2200[1855 ; 2831] \mathrm{Aa}$ & $2428[2234 ; 3088] \mathrm{Aa}$ \\
\hline \multirow[t]{2}{*}{$\operatorname{IRVP}^{1 *}\left(\right.$ dina.s. $\left.\mathrm{cm}^{-5} \cdot \mathrm{m}^{-2}\right)$} & G1 & $154[128 ; 190]$ & $164[128 ; 191]$ & $131[110 ; 171]$ & $142[123 ; 202]$ \\
\hline & G2 & $166[118 ; 198]$ & $172[165 ; 192]$ & $188[121 ; 236]$ & $169[125 ; 256]$ \\
\hline \multirow[t]{3}{*}{ ITSVE $^{2 *}\left(\mathrm{~g} \cdot \mathrm{m} \cdot \mathrm{m}^{-2}\right)$} & G1 & $43,8 \pm 13,0$ & $43,5 \pm 13,9$ & $43,7 \pm 12,3$ & $43,8 \pm 11,9$ \\
\hline & G2 & $42,7 \pm 18,8$ & $44,2 \pm 14,6$ & $44,9 \pm 15,0$ & $40,8 \pm 12,9$ \\
\hline & G3 & $44,9 \pm 16,5$ & $42,7 \pm 16,3$ & $41,6 \pm 12,5$ & $37,1 \pm 10,8$ \\
\hline \multirow[t]{3}{*}{ ITSVD $^{2 *}\left(\mathrm{~g} \cdot \mathrm{m} \cdot \mathrm{m}^{-2}\right)$} & G1 & $4,4 \pm 1,9$ & $4,6 \pm 2,0$ & $5,2 \pm 1,5$ & $5,1 \pm 1,6$ \\
\hline & G2 & $4,3 \pm 2,0$ & $3,7 \pm 1,5$ & $4,3 \pm 1,5$ & $3,8 \pm 1,0$ \\
\hline & G3 & $4,1 \pm 1,6$ & $3,8 \pm 1,4$ & $3,9 \pm 1,2$ & $3,2 \pm 1,4$ \\
\hline
\end{tabular}

${ }^{1}$ Mediana, $1^{\circ}$ e $3^{\circ}$ quartis; ${ }^{2}$ Média $\pm \mathrm{DP} ;{ }^{3}$ Grupos dentro de cada momento, seguidos de mesma letra maiúscula, não diferem estatisticamente ( $\left.\mathrm{p}>0,05\right) ;{ }^{4}$ Momentos dentro de cada grupo, seguidos de mesma letra minúscula, não diferem estatisticamente $(p>0,05) ;{ }^{*} p>0,05(e n t r e ~ g r u p o s$, dentro de cada momento e entre momentos, dentro de cada grupo); Obs: $\mathrm{A}>\mathrm{B}$; $\mathrm{a}>\mathrm{b}>\mathrm{c}$

Tabela IV - Valores da Saturação de Pulso de Oxigênio $\left(\mathrm{SpO}_{2}\right)$, Pressão Expiratória Final de $\mathrm{CO}_{2}\left(\mathrm{P}_{\mathrm{ET}} \mathrm{CO}_{2}\right)$, do Hematócrito $(\mathrm{Ht})$ e da Temperatura Esofágica $(T)$ nos Momentos e Grupos Estudados

\begin{tabular}{|c|c|c|c|c|c|}
\hline \multirow[t]{2}{*}{ Atributos } & \multirow[t]{2}{*}{ Grupos } & \multicolumn{4}{|c|}{ Momentos } \\
\hline & & $\mathrm{M}_{1}$ & $\mathrm{M}_{2}$ & $\mathrm{M}_{3}$ & $\mathrm{M}_{4}$ \\
\hline \multirow[t]{3}{*}{$\mathrm{SpO}_{2}{ }^{1 *}(\%)$} & G1 & $99,0[99,0 ; 99,0]$ & $99,0[99,0 ; 99,0]$ & $98,5[98,0 ; 99,0]$ & $98,0[98,0 ; 99,0]$ \\
\hline & G2 & $99,0[99,0 ; 99,0]$ & $99,0[99,0 ; 99,0]$ & $99,0[98,0 ; 99,0]$ & $99,0[98,0 ; 99,0]$ \\
\hline & G3 & $99,0[98,5 ; 99,5]$ & $99,0[99,0 ; 99,5]$ & $99,0[98,5 ; 99,0]$ & $99,0[98,0 ; 99,0]$ \\
\hline \multirow[t]{3}{*}{$\mathrm{P}_{\mathrm{ET}} \mathrm{CO}_{2}{ }^{2 *}(\mathrm{mmHg})$} & G1 & $37,2 \pm 3,8$ & $38,1 \pm 4,7$ & $39,2 \pm 3,6$ & $40,3 \pm 4,5$ \\
\hline & G2 & $36,4 \pm 5,6$ & $37,6 \pm 6,4$ & $37,7 \pm 5,9$ & $39,7 \pm 6,8$ \\
\hline & G3 & $36,2 \pm 5,4$ & $36,8 \pm 6,5$ & $37,2 \pm 6,7$ & $37,1 \pm 7,1$ \\
\hline \multirow[t]{3}{*}{$\mathrm{Ht}^{1 *}(\%)$} & G1 & $39,5[36,0 ; 43,0]$ & $40,0[36,5 ; 44,0]$ & $41,0[37,0 ; 43,5]$ & $41,0[37,0 ; 42,5]$ \\
\hline & G2 & $41,0[38,5 ; 44,5]$ & $42,0[36,0 ; 46,0]$ & $41,5[39,0 ; 46,5]$ & $43,5[40,0 ; 46,0]$ \\
\hline & G3 & $39,5[36,5 ; 42,0]$ & $41,0[35,5 ; 44,0]$ & $41,5[36,0 ; 44,0]$ & $41,5[36,0 ; 45,0]$ \\
\hline \multirow[t]{3}{*}{$\mathrm{T}^{1 *}\left({ }^{\circ} \mathrm{C}\right)$} & G1 & $38,0[37,3 ; 38,3]$ & $37,8[36,9 ; 38,7]$ & $38,1[37,5 ; 38,9]$ & $38,4[37,6 ; 39,0]$ \\
\hline & G2 & $37,9[37,0 ; 38,8]$ & $37,9[37,1 ; 39,1]$ & $37,8[37,1 ; 39,4]$ & $38,1[37,3 ; 39,3]$ \\
\hline & G3 & $36,9[36,4 ; 38,3]$ & $37,2[36,2 ; 38,1]$ & $37,0[36,6 ; 38,7]$ & $37,3[36,5 ; 38,7]$ \\
\hline
\end{tabular}

${ }^{1}$ Mediana, $1^{\circ}$ e $3^{\circ}$ quartis; ${ }^{2}$ Média \pm DP; * $p>0,05$ (entre grupos, dentro de cada momento e entre momentos, dentro de cada grupo) 


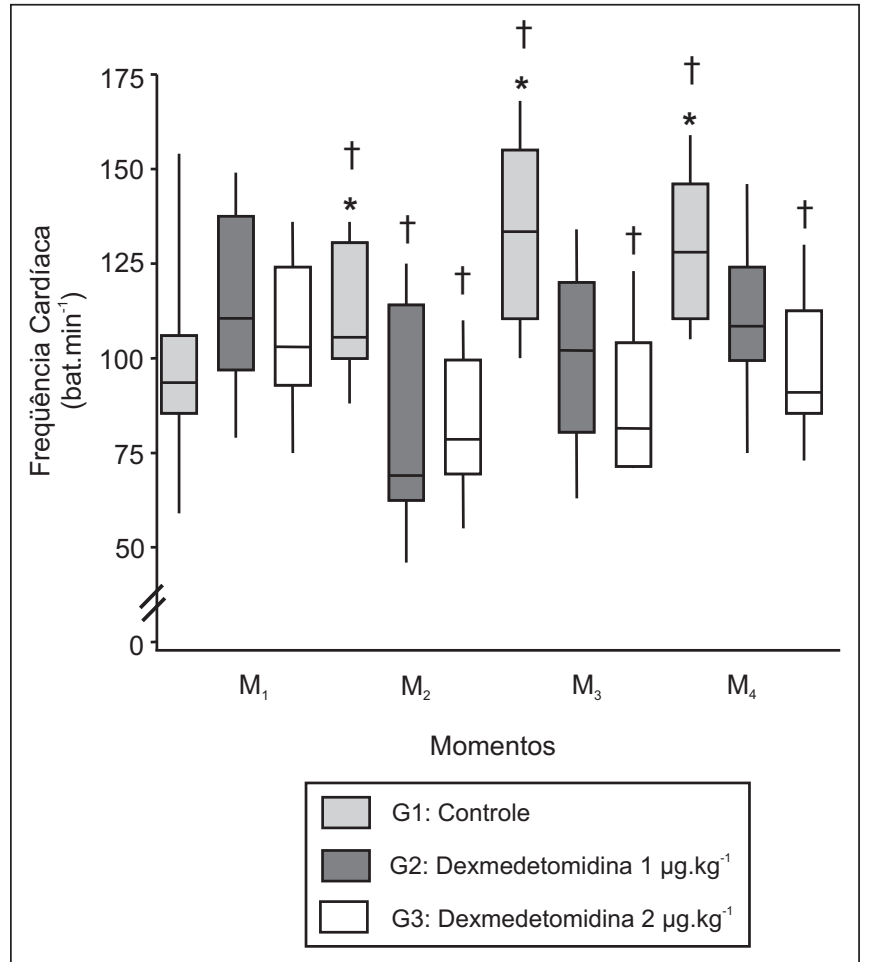

Figura 1 - Freqüência Cardíaca. Mediana, $1^{\circ}$ e $3^{\circ}$ Quartis, Valor Máximo e Mínimo obtidos nos Grupos e Momentos Estudados

${ }^{*} p<0,05$ entre grupos, dentro de cada momento

$\dagger p<0,05$ entre momentos, dentro de cada grupo

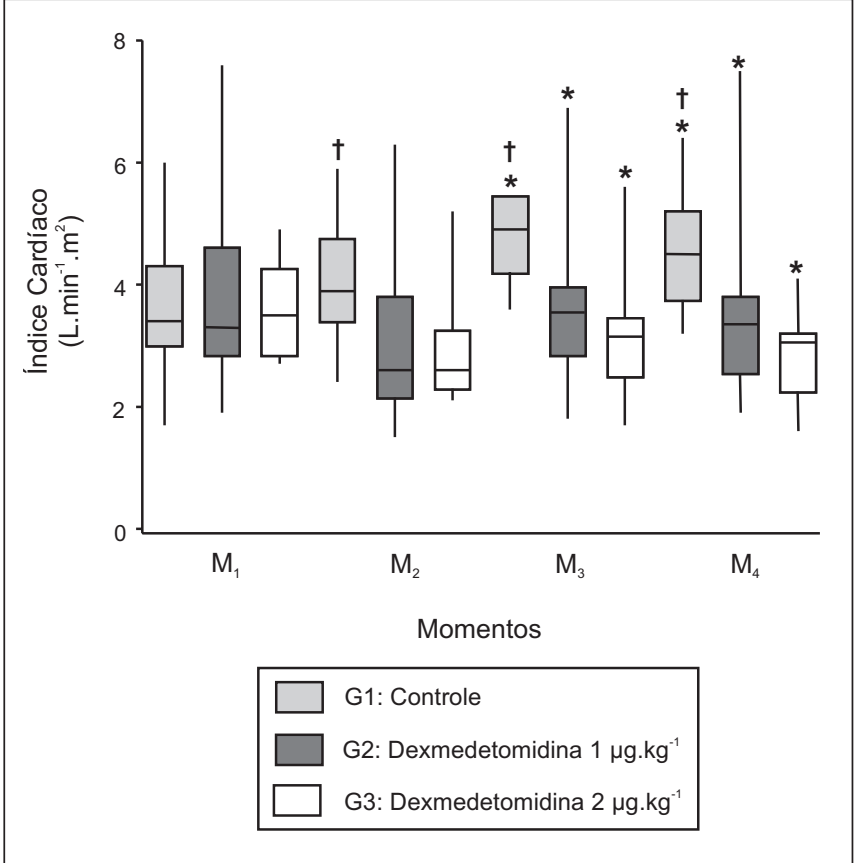

Figura 3 - Índice Cardíaco. Mediana, $1^{\circ}$ e $3^{\circ}$ Quartis, Valor Máximo e Mínimo obtidos nos Grupos e Momentos Estudados

* $p<0,05$ entre grupos, dentro de cada momento

$\dagger p<0,05$ entre momentos, dentro de cada grupo

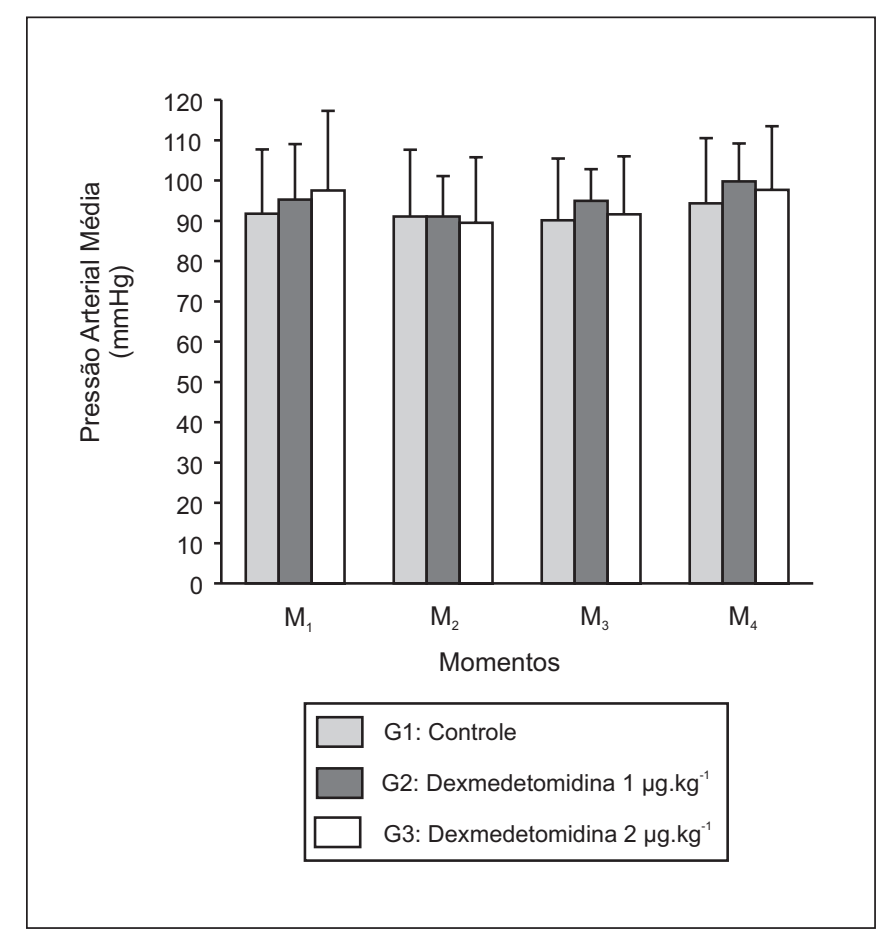

Figura 2 - Pressão Arterial Média. Média e Desvio Padrão dos Valores Observados em cada Momento nos Três Grupos Estudados $(p>0,05)$

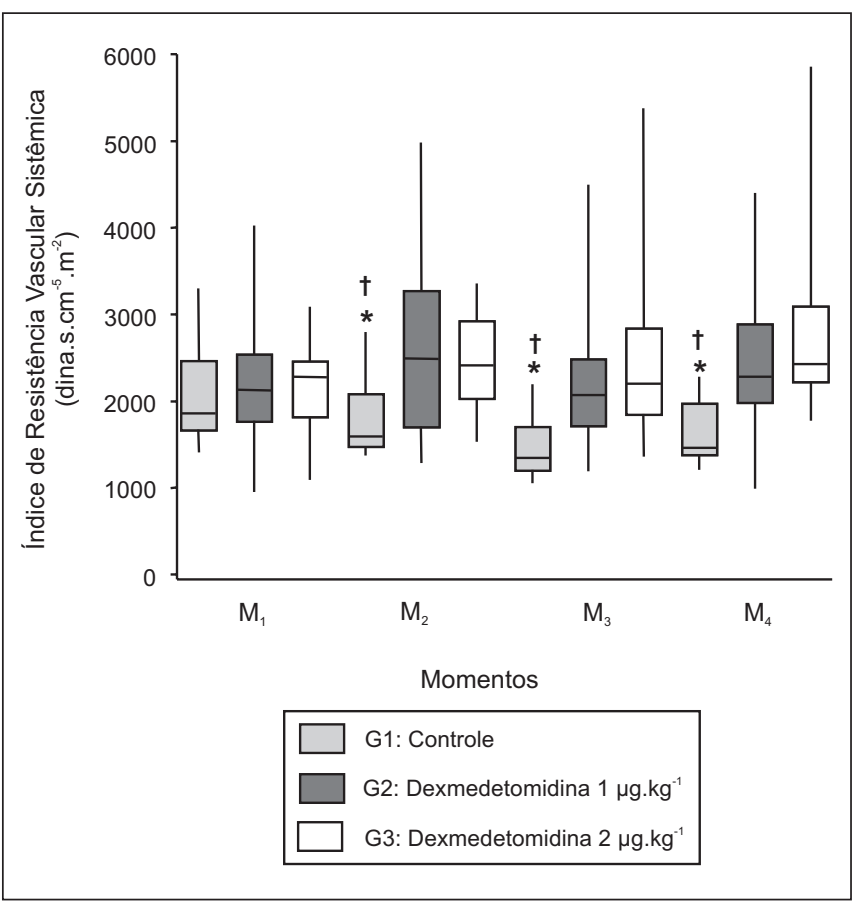

Figura 4 - Índice de Resistência Vascular Sistêmica. Mediana, $1^{\circ} \mathrm{e}$ $3^{\circ}$ Quartis, Valor Máximo e Mínimo obtidos nos Grupos e Momentos Estudados

${ }^{*} p<0,05$ entre grupos, dentro de cada momento $\dagger p<0,05$ entre momentos, dentro de cada grupo

Revista Brasileira de Anestesiologia Vol. 53, No 6, Novembro - Dezembro, 2003 
AFC foi menor no $\mathrm{G} 2$ e G3, em relação ao G1, nos momentos $M_{2}, M_{3}$ e $M_{4}(p<0,05)$. Não houve diferença significativa entre os grupos 2 e 3 . Nos grupos, observamos que no G2 a FC diminuiu no $\mathrm{M}_{2}(p<0,05)$, retornando aos valores do momento controle nos momentos $\mathrm{M}_{3}$ e $\mathrm{M}_{4}$. No G3 a FC diminuiu no $\mathrm{M}_{2}$ e manteve-se abaixo dos valores do momento controle nos momentos $\mathrm{M}_{3}$ e $\mathrm{M}_{4}(\mathrm{p}<0,05)$. O G1 apresentou aumento progressivo da FC $(p<0,05)$ (Figura 1$)$.

A PAM não apresentou diferença significativa entre os grupos, nem nos diferentes momentos de cada grupo $(p>0,05)$ (Figura 2).

APAP não apresentou diferença significativa entre os grupos $(p>0,05)$. Nos grupos, observamos que no G1 a PAP foi maior nos momentos $M_{3}$ e $M_{4}$ em relação aos momentos $M_{1}$ e $M_{2}$ $(p<0,05)$, enquanto não houve diferença nos grupos $G 2$ e G3 (Tabela II).

O IC, nos momentos $\mathrm{M}_{3}$ e $\mathrm{M}_{4}$, foi menor no $\mathrm{G} 2$ e G3 em relação ao $G 1$ e no $G 3$ em relação ao $G 2(p<0,05)$. Nos grupos, no $\mathrm{G} 1$ houve aumento do IC nos momentos $\mathrm{M}_{2}, \mathrm{M}_{3}$ e $\mathrm{M}_{4}$ em relação ao momento controle $(p<0,05)$, enquanto não se observou diferença no G2 e G3 (Figura 3).

O IRVS foi menor no $\mathrm{G} 1$ nos momentos $\mathrm{M}_{2}, \mathrm{M}_{3}$ e $\mathrm{M}_{4}$ em relação ao G2 e G3 ( $p<0,05)$. Não houve diferença entre G2 e G3. Nos grupos, no $\mathrm{G} 1$ houve redução do IRVS nos momentos $M_{2}, M_{3}$ e $M_{4}$ em relação ao $M_{1}(p<0,05)$, enquanto no $G 2$ e G3 não se observou diferença entre os momentos estudados (Figura 4).

As variáveis PAPO, PAD, IS, IRVP, ITSVD, ITSVE, Ht, $\mathrm{P}_{\mathrm{ET}} \mathrm{CO}_{2}, \mathrm{SpO}_{2}$ e T não apresentaram diferença entre os grupos, nem nos diferentes momentos de cada grupo $(p>0,05)$.

\section{DISCUSSÃO}

No momento controle, os valores das variáveis hemodinâmicas estudadas permaneceram dentro da faixa de normalidade para cães em repouso ${ }^{20}$, tendo sido o resultado da boa estabilidade hemodinâmica promovida pela escolha da técnica anestésica balanceada ${ }^{21}$ e da adequada hidratação realizada nos animais.

Assim como neste experimento, vários trabalhos relataram redução da FC após injeção de dexmedetomidina ${ }^{8,22-24}$. Bloor e col. ${ }^{25}$ descreveram diminuição da FC após a injeção de dexmedetomidina em cães, na dose de $20 \mu \mathrm{g} \cdot \mathrm{kg}^{-1} \mathrm{em}$ intervalo de 2 minutos, precedida de aumento da PAM. Dycke col. ${ }^{26}$ observaram, também, aumento da PAM e redução da FC após injeção intravenosa de dexmedetomidina em voluntários, na dose de $2 \mu \mathrm{g} . \mathrm{kg}^{-1}$ em intervalo de 5 minutos; porém, essas alterações não ocorreram quando foi utilizada a via muscular. Esses autores sugeriram que um dos mecanismos envolvidos na redução da FC após a injeção de dexmedetomidina é a ativação do barorreflexo e a injeção lenta da droga tenderia a minimizá-la. Observamos, neste trabaIho, redução da FC que não foi precedida de aumento da PAM. Vários outros autores também observaram diminuição da FC sem alteração da PAM após injeção de dexmedetomidina ${ }^{27-29}$. Além da ativação do barorreflexo, outros fatores podem estar envolvidos na redução da FC observada após a injeção da dexmedetomidina, como aumento da atividade do sistema nervoso parassimpático por estimulação do núcleo do trato solitário ${ }^{30,31}$, diminuição central da atividade do sistema nervoso simpático (SNS) ${ }^{32}$ ou inibição da liberação de catecolaminas nas terminações nervosas do SNS $7,27,33$. Observamos que a redução da FC foi maior e mais duradoura com a maior dose da dexmedetomidina, confirmando ser esta alteração dose-dependente ${ }^{34}$. No grupo controle, a FC apresentou aumento durante o experimento, resultado, provavelmente, da ativação do barorreflexo pelo isoflurano ou de hiperatividade do SNS devido à manutenção da anestesia com 0,6 CAM de isoflurano, o que não aconteceu nos grupos que receberam dexmedetomidina, já que esta droga diminui a CAM do isoflurano em até $70 \%{ }^{6}$.

Os receptores $\alpha_{2}$-adrenérgicos são classificados, conforme sua ação farmacológica, em $\alpha_{2} A, \alpha_{2} B$ e $\alpha_{2} C$. Os receptores $\alpha_{2}$ pré-juncionais são principalmente os $\alpha_{2} A$, embora possam estar presentes também os $\alpha_{2} \mathrm{C}^{35}$. Eles inibem a liberação de noradrenalina nas terminações nervosas simpáticas e nos neurônios noradrenérgicos no sistema nervoso central ${ }^{36}$. Todos os três subtipos de receptores podem ser identificados no músculo liso dos vasos e promovem vasoconstrição, sendo que os $\alpha_{2} \mathrm{C}$ estão localizados principalmente nas veias ${ }^{36}$. A dexmedetomidina também se liga aos receptores imidazólicos e é provável que algumas alterações hemodinâmicas observadas após a injeção dessa droga também sejam mediadas por esses receptores ${ }^{37}$.

Vários autores observaram que a dexmedetomidina promove alteração bifásica da PAM, ocorrendo aumento inicial e posterior redução ${ }^{8,26}$. Ebert e col. ${ }^{9}$ relataram que concentrações plasmáticas da dexmedetomidina abaixo de $1,2 \mathrm{ng} \cdot \mathrm{ml}^{-1}$ produzem redução da PAM, enquanto concentrações acima de $1,9 \mathrm{ng} \cdot \mathrm{ml}^{-1}$ desencadeiam aumento progressivo da PAM, por prevalecer a ação dessa droga sobre os receptores $\alpha_{2}$ pós-juncionais. Não observamos, neste experimento, alteração significativa da PAM após injeção da dexmedetomidina. Vários autores relataram que a injeção da dexmedetomidina, no cão, promove predominantemente aumento da PAM $24,38,39$, não reproduzido, contudo, neste experimento, provavelmente por termos utilizado, ao contrário de outros autores, doses menores de dexmedetomidina, semelhantes às que são utilizadas na prática clínica, ou, também, por resposta vasoconstritora promovida pela droga ter sido bloqueada pelo isoflurano ${ }^{40}$.

Embora possamos observar diminuição da PAM com o uso da dexmedetomidina por sua ação sobre os receptores $\alpha_{2}$ pré-juncionais, há, paradoxalmente, aumento da resistência vascular sistêmica após utilização de doses elevadas dessa droga $^{23}$. Diferentemente de outros autores ${ }^{23-25}$, não observamos aumento do IRVS nos cães que receberam dexmedetomidina. Dois fatores podem ser responsáveis por essa diferença de resultados. Primeiro, a dose de dexmedetomidina utilizada neste experimento foi menor que as utilizadas em experimentos prévios, e, segundo, o isoflurano, por ser um agente vasodilatador ${ }^{41}$, contrabalançou a resposta vasoconstritiva da dexmedetomidina. Assim, ainda que não te- 
nhamos observado aumento do IRVS com o uso da dexmedetomidina, essa droga foi capaz de impedir a redução do IRVS observada no grupo controle e ausente nos grupos tratados.

Em trabalhos prévios, a dexmedetomidina foi capaz de reduziro IC, de forma dose-dependente, por reduzir a FC, aumentar a pós-carga ${ }^{9,24}$ ou comprometer a contratilidade miocárdica por diminuir o fluxo coronariano ${ }^{23}$. As doses de dexmedetomidina utilizadas neste experimento não produziram alterações significativas do IC, mas impediram o aumento progressivo e significativo do IC observado no grupo controle, por ter reduzido a FC, ter impedido a redução da pós-carga produzida pelo isoflurano e por ter inibido a resposta autonômica desencadeada, provavelmente, por plano anestésico superficial no $\mathrm{G} 1$.

APAP apresentou aumento no G1, que não foi observado nos grupos que receberam dexmedetomidina. Esse aumento na PAP foi resultado da elevação da FC observada no grupo controle.

Adexmedetomidina não promoveu, neste experimento, alterações na PAPO, na PAD, no IS, no IRVP, no ITSVD e no ITSVE, confirmando os resultados de experimentos prévios 10,15,34, em que evidenciou-se que, com doses baixas de dexmedetomidina, próximas das utilizadas na prática clínica, há sedação, analgesia, diminuição da concentração plasmática de noradrenalina e redução da FC sem, contudo, alterar os parâmetros hemodinâmicos de forma importante.

Assim, concluímos que a dexmedetomidina no cão, nas condições experimentais empregadas, promoveu diminuição dose-dependente da FC, não alterou a PAM, impediu o aumento do IC observado no grupo controle e inibiu a redução do IRVS promovida pelo isoflurano, mostrando ser fármaco seguro e capaz de promover boa estabilidade hemodinâmica.

\section{Cardiovascular Effects of Two Dexmedetomidine Doses. Experimental Study in Dogs}

Nivaldo Ribeiro Villela, M.D.; Paulo do Nascimento Júnior, TSA, M.D.; Lídia Raquel de Carvalho, M.D.

\section{INTRODUCTION}

Sympathetic nervous system (SNS) activation during different anesthetic and surgical procedures may lead to hemodynamic instability and metabolic changes in coronary disease patients ${ }^{1}$, increasing the incidence of ischemia, myocardial infarction or severe arrhythmias ${ }^{2}$. Aiming at minimizing this hyperdynamic cardiovascular response, there is a growing interest in the use of $\alpha_{2}$-adrenergic agonists in the intraoperative period as anesthetic adjuvants ${ }^{3}$.
Dexmedetomidine, medetomindine's dextrogyrous enantiomer, is a super-selective $\alpha_{2}$-adrenergic agonist with selectivity ratio between receptors of $\alpha_{2}: \alpha_{1} 1600: 1^{4}$, having shown major sedative and analgesic action in experimental studies, decreasing anesthetics and opioids consumption during anesthesia ${ }^{6}$.

In healthy volunteers, dexmedetomidine decreases catecholamine plasma concentrations in up to $90 \%^{7,8}$, promoting sedation, hypnosis and analgesia ${ }^{9}$. In surgical patients, dexmedetomidine decreases anesthetics ${ }^{10-12}$ and opioids ${ }^{13,14}$ consumption, maintains better hemodynamic stability ${ }^{10,15}$ and decreases postoperative analgesics and sedatives consumption ${ }^{16}$.

Dexmedetomidine cardiovascular effects are primarily characterized by mean blood pressure (MBP) and heart rate (HR) decrease. The intensity of such changes depends on the dose, administration route and sympathetic tone of the animal receiving the drug ${ }^{17}$. Bradycardia and arterial hypotension are adverse effects observed with dexmedetomidine ${ }^{18}$ and have been a reason of concern among anesthesiologists ${ }^{19}$.

There are few experimental studies in the literature assessing dexmedetomidine in conditions similar to those practiced in operating rooms. So, our study aimed at evaluating dexmedetomidine hemodynamic cardiovascular effects in anesthetized dogs, using two different doses, but similar to those used in Anesthesiology.

\section{METHODS}

After the Animal Experiment Ethics Committee, Faculdade de Medicina, Botucatu, UNESP approval, 36 adult mixed-breed dogs of both genders, weighing 18 to $30 \mathrm{~kg}$ were included in the study.

\section{Anesthetic-Surgical Preparation}

After 12-hour fast, but with free access to water, animals were anesthetized with propofol $\left(6 \mathrm{mg} \cdot \mathrm{kg}^{-1}\right)$ and fentanyl ( 5 $\left.\mu \mathrm{g} . \mathrm{kg}^{-1}\right)$. After tracheal intubation, animals were mechanically ventilated with oxygen $\left(0.8 \mathrm{~L}_{\mathrm{min}} \mathrm{m}^{-1}\right)$ and air $(1.2$ L. $\left.\mathrm{min}^{-1}\right)$, tidal volume of $20 \mathrm{ml}^{\mathrm{kg}} \mathrm{kg}^{-1}$ and respiratory rate of 12 to 16 movements per minute, aiming at maintaining $\mathrm{CO}_{2}$ end expiratory pressure between 35 and $45 \mathrm{mmHg}$. Isoflurane administration was then started and adjusted to maintain end expiratory concentration at 1.7 MAC. Right femoral vein was dissected and catheterized for hydration with lactated Ringer's solution (18 ml. $\left.\mathrm{kg}^{-1} \cdot \mathrm{h}^{-1}\right)$ and rocuronium (0.6 mg. $\mathrm{kg}^{-1}$ and continuous $10 \mu \mathrm{g} \cdot \mathrm{kg}^{-1} \cdot \mathrm{min}^{-1}$ ) was administered. Then, left femoral artery was dissected and catheterized for continuous MBP readings, left femoral vein was dissected and catheterized for blood collection for hematocrit measurement $(\mathrm{Ht})$ and right jugular vein was dissected for insertion of 7F Swan-Ganz catheter in the pulmonary artery for cardiac output assessment by thermodilution, right atrium mean blood pressure reading (RAP), pulmonary artery mean pressure reading (PAP) 
and pulmonary capillary wedge pressure reading (PCWP). Animals' temperature was maintained with heated air flowing on the ventral surface $\left(38\right.$ to $42^{\circ} \mathrm{C}$ ) and warming of injected solutions. Temperature was monitored with a sensor placed in the esophagus.

After surgical preparation completion, wounds were infiltrated with $0.2 \%$ ropivacaine, isoflurane expired concentration was decreased to 0.6 MAC and lactated Ringer's solution volume was decreased to $6 \mathrm{ml} \cdot \mathrm{kg}^{-1} \cdot \mathrm{h}^{-1}$. A hemodynamic stabilization period of 30 minutes was allowed before the first study evaluation (control).

\section{Studied Groups}

After the control moment, animals were randomly and double-blindly distributed in 3 groups of 12 dogs each:

G1 ( $n=12)$ : was given $20 \mathrm{ml}$ of $0.9 \%$ saline solution injected in 10 minutes, followed by $20 \mathrm{ml}$ of the same solution infused in 1 hour.

G2 ( $n=12)$ : was given $20 \mathrm{ml}$ of $0.9 \%$ saline with 1 $\mu \mathrm{g} . \mathrm{kg}^{-1}$ dexmedetomidine injected in 10 minutes, followed by the same dexmedetomidine dose (1 $\left.\mu \mathrm{g} \cdot \mathrm{kg}^{-1}\right)$ infused in 1 hour.

G3 $(n=12)$ : was given $20 \mathrm{ml}$ of $0.9 \%$ saline solution with 2 $\mu \mathrm{g} . \mathrm{kg}^{-1}$ dexmedetomidine injected in 10 minutes, followed by the same dexmedetomidine dose $\left(2 \mu \mathrm{g} . \mathrm{kg}^{-1}\right)$ infused in 1 hour.

\section{Studied Attributes}

The following attributes were evaluated: to control study homogeneity-length, weight, body surface area (BSA), gender, hematocrit $(\mathrm{Ht}), \mathrm{P}_{\mathrm{ET}} \mathrm{CO}_{2}$, oxygen pulse saturation $\left(\mathrm{SpO}_{2}\right)$ and esophageal temperature $(\mathrm{T})$; to meet experiment objectives $\mathrm{HR}, \mathrm{MBP}, \mathrm{PAP}, \mathrm{RAP}, \mathrm{PCWP}$, cardiac index $(\mathrm{Cl})$, systolic index (SI), systemic vascular resistance index (SVRI), pulmonary vascular resistance index (PVRI), left ventricle systolic work index (LVSWI) and right ventricle systolic work index (RVSWI).

\section{Evaluated Moments}

Attributes were evaluated in 4 moments:

$\mathrm{M}_{1}$ (control): immediately after anesthetic stabilization period;

$\mathrm{M}_{2}$ : after initial $20 \mathrm{ml}$ injection of the studied solution in 10 minutes, coincident with beginning of continuous injection of the studied solution in 1 hour;

$M_{3}$ : 60 minutes after $M_{2}$, coincident with end of studied solution injection in 1 hour;

$\mathrm{M}_{4}: 60$ minutes after $\mathrm{M}_{3}$.

\section{Statistical Analysis}

Profile Analysis was used for variables with normal distribution and variance homogeneity, followed by Tukey's test for multiple comparisons. Friedman test was used for variables without normal distribution or variance homogeneity, for comparisons among moments, and Kruskall-Wallis test was used to compare groups, followed by multiple comparisons test. Analysis of Variance was used for demographics. Fisher Exact test was used to analyze gender frequency. Significance level was $5 \%$.

\section{RESULTS}

Groups were homogeneous in weight, length, body surface area (BSA) and gender (Table I).

Table I - Weight, Length, Body Surface Area (BSA) and Gender Distribution in each Studied Group

\begin{tabular}{lccc}
\hline Groups & $\begin{array}{c}\mathrm{G} 1 \\
(\mathrm{n}=12)\end{array}$ & $\begin{array}{c}\mathrm{G} 2 \\
(\mathrm{n}=12)\end{array}$ & $\begin{array}{c}\mathrm{G} 3 \\
(\mathrm{n}=12)\end{array}$ \\
\hline Weight $(\mathrm{kg})^{*}$ & $24.3 \pm 4.2$ & $21.9 \pm 3.9$ & $23.9 \pm 3.7$ \\
Length $(\mathrm{cm})^{*}$ & $114.7 \pm 6.9$ & $107.3 \pm 8.0$ & $114.8 \pm 10.1$ \\
BSA $\left(\mathrm{m}^{2}\right)^{*}$ & $0.90 \pm 0.09$ & $0.85 \pm 0.09$ & $0.86 \pm 0.11$ \\
Gender & & & \\
\multicolumn{1}{c}{ Male } & 9 & 10 & 12 \\
Female & 3 & 2 & 0 \\
\hline
\end{tabular}

* Values expressed in Mean \pm SD

Hemodynamic variables $\left(\mathrm{Ht}, \mathrm{P}_{\mathrm{ET}} \mathrm{CO}_{2}\right.$ and $\mathrm{SpO}_{2}$ ) are shown in tables II, III, IV, and figures 1, 2, 3, 4.

HR was lower in $\mathrm{G} 2$ and $\mathrm{G} 3$ as compared to $\mathrm{G} 1$ at moments $M_{2}, M_{3}$ and $M_{4}(p<0.05)$. There were no significant differences between $\mathrm{G} 2$ and $\mathrm{G} 3$. HR has decreased at $\mathrm{M}_{2}(p<0.05)$ in $\mathrm{G} 2$, returning to baseline values at $M_{3}$ and $M_{4}$. In $G 3, H R$ has decreased at $M_{2}$ and remained below baseline values at $M_{3}$ and $M_{4}(p<0.05)$. HR has progressively increased in $G 1$ ( $p$ $<0.05)$ (Figure 1).

There were no significant differences in MBP among groups and at different moments within groups $(p>0.05)$ (Figure 2$)$.

There were no significant differences in PAP among groups $(p>0.05)$. Within groups, it has been observed that in $G 1$, PAP was higher at $M_{3}$ and $M_{4}$ as compared to $M_{1}$ and $M_{2}(p<$ 0.05 ), while there have been no differences in $\mathrm{G} 2$ and $\mathrm{G} 3$ (Table II).

Cl was lower in $\mathrm{G} 2$ and $\mathrm{G} 3$ at $\mathrm{M}_{3}$ and $\mathrm{M}_{4}$ as compared to $\mathrm{G} 1$, and in $\mathrm{G} 3$ as compared to $\mathrm{G} 2(\mathrm{p}<0.05)$. Within groups, there has been $\mathrm{Cl}$ increase in $\mathrm{G} 1$ at $\mathrm{M}_{2}, \mathrm{M}_{3}$ and $\mathrm{M}_{4}$ as compared to control moment $(p<0.05)$, while there have been no differences in $\mathrm{G} 2$ and $\mathrm{G} 3$ (Figure 3).

SVRI was lower at $\mathrm{G} 1$ in $\mathrm{M}_{2}, \mathrm{M}_{3}$ and $\mathrm{M}_{4}$ as compared to $\mathrm{G} 2$ and $\mathrm{G} 3(p<0.05)$. There have been no differences between $G 2$ and $G 3$. Within groups, there has been SVRI decrease in $G 1$ at $M_{2}, M_{3}$ and $M_{4}$ as compared to $M_{1}(p<0.05)$, while there have been no differences at studied moments in $\mathrm{G} 2$ and $\mathrm{G} 3$ (Figure 4).

There have been no differences in PCWP, RAP, SI, RVSWI, LVSWI, $\mathrm{Ht}, \mathrm{P}_{\mathrm{ET}} \mathrm{CO}_{2}, \mathrm{SpO}_{2}$ and $\mathrm{T}$ between groups or at different moments within each group $(p>0.05)$. 
Table II - Heart Rate (HR), Mean Blood Pressure (MBP), Pulmonary Artery Mean Pressure (PAP), Mean Right Atrium Pressure (RAP), Pulmonary Capillary Wedge Pressure (PCWP) and Cardiac Index (CI) within Studied Moments and Groups

\begin{tabular}{|c|c|c|c|c|c|}
\hline \multirow[t]{2}{*}{ Attributes } & \multirow[t]{2}{*}{ Groups } & \multicolumn{4}{|c|}{ Moments } \\
\hline & & $\mathrm{M}_{1}$ & $\mathrm{M}_{2}$ & $M_{3}$ & $\mathrm{M}_{4}$ \\
\hline \multirow[t]{3}{*}{$\mathrm{HR}^{1}\left(\right.$ beat. $\left.\mathrm{min}^{-1}\right)$} & $\mathrm{G} 1$ & $93[85 ; 106] \mathrm{A}^{3} \mathrm{c}^{4}$ & $105[100 ; 130] \mathrm{Ab}$ & $133[110 ; 155] \mathrm{Aa}$ & $128[110 ; 146] \mathrm{Aa}$ \\
\hline & $\mathrm{G} 2$ & $110[97 ; 137] \mathrm{Aa}$ & $69[62 ; 114] \mathrm{Bb}$ & $102[80 ; 120] \mathrm{Ba}$ & $108[99 ; 124] \mathrm{Ba}$ \\
\hline & G3 & $103[93 ; 124] \mathrm{Aa}$ & 78 [69; 99] Bb & $81[71 ; 104] \mathrm{Bb}$ & $91[85 ; 112] \mathrm{Bb}$ \\
\hline \multirow[t]{3}{*}{$\mathrm{MBP}^{2 *}(\mathrm{mmHg})$} & $\mathrm{G} 1$ & $91.8 \pm 16.0$ & $91.1 \pm 16.6$ & $90.1 \pm 15.4$ & $94.3 \pm 16.2$ \\
\hline & G2 & $95.3 \pm 13.8$ & $91.1 \pm 10.0$ & $94.9 \pm 7.9$ & $99.8 \pm 9.4$ \\
\hline & G3 & $97.5 \pm 19.7$ & $89.5 \pm 16.3$ & $91.6 \pm 14.4$ & $97.7 \pm 15.8$ \\
\hline \multirow[t]{3}{*}{$\operatorname{PAP}^{2}(\mathrm{mmHg})$} & G1 & $11.7 \pm 2.9 \mathrm{Ab}$ & $12.3 \pm 2.6 \mathrm{Aab}$ & $13.2 \pm 2.4 \mathrm{Aa}$ & $13.6 \pm 2.1 \mathrm{Aa}$ \\
\hline & $\mathrm{G} 2$ & $12.0 \pm 1.5 \mathrm{Aa}$ & $12.4 \pm 1.3 \mathrm{Aa}$ & $12.6 \pm 2.3 \mathrm{Aa}$ & $12.8 \pm 2.0 \mathrm{Aa}$ \\
\hline & G3 & $10.8 \pm 2.6 \mathrm{Aa}$ & $11.2 \pm 3.5 \mathrm{Aa}$ & $11.8 \pm 2.9 \mathrm{Aa}$ & $10.9 \pm 3.5 \mathrm{Aa}$ \\
\hline \multirow[t]{3}{*}{$\operatorname{RAP}^{1 *}(\mathrm{mmHg})$} & $\mathrm{G} 1$ & $3.5[1.5 ; 5.0]$ & $3.0[2.5 ; 4.5]$ & $3.0[2.5 ; 4.0]$ & $3.5[2.5 ; 4.0]$ \\
\hline & $\mathrm{G} 2$ & $2.5[1.0 ; 4.0]$ & $5.0[3.0 ; 7.0]$ & $5.0[3.0 ; 7.0]$ & $4.0[3.0 ; 4.5]$ \\
\hline & G3 & $2.0[1.0 ; 4.0]$ & $3.0[1.5 ; 5.5]$ & $3.0[2.0 ; 5.5]$ & $2.5[1.5 ; 5.0]$ \\
\hline \multirow[t]{3}{*}{$\mathrm{PCWP}^{1}{ }^{*}(\mathrm{mmHg})$} & $\mathrm{G} 1$ & $4.5[3.5 ; 7.0]$ & $4.0[2.0 ; 6.5]$ & $5.0[3.5 ; 6.0]$ & $5.0[3.5 ; 6.0]$ \\
\hline & $\mathrm{G} 2$ & $3.5[3.0 ; 5.0]$ & $6.0[6.0 ; 7.0]$ & $5.0[4.0 ; 6.0]$ & $5.0[4.0 ; 7.0]$ \\
\hline & G3 & $4.5[1.5 ; 5.5]$ & $5.5[2.5 ; 7.5]$ & $6.0[3.5 ; 8.0]$ & $5.0[3.0 ; 7.0]$ \\
\hline \multirow[t]{3}{*}{$\mathrm{Cl}^{1}\left(\right.$ L. $\left.\min \cdot \mathrm{m}^{-2}\right)$} & $\mathrm{G} 1$ & $3.4[3.0 ; 4.3] \mathrm{Ac}$ & $3.9[3.4 ; 4.8] \mathrm{Ab}$ & $4.9[4.2 ; 5.5] \mathrm{Aa}$ & $4.5[3.8 ; 5.2] \mathrm{Aa}$ \\
\hline & $\mathrm{G} 2$ & $3.3[2.9 ; 4.6] \mathrm{Aa}$ & $2.6[2.2 ; 3.8] \mathrm{Aa}$ & $3.6[3.6 ; 2.9] \mathrm{Ba}$ & $3.4[2.6 ; 3.8] \mathrm{Ba}$ \\
\hline & G3 & $3.5[2.9 ; 4.3] \mathrm{Aa}$ & $2.6[2.3 ; 3.3] \mathrm{Aa}$ & $3.2[2.5 ; 3.5] \mathrm{Ca}$ & $3.1[2.3 ; 3.2] \mathrm{Ca}$ \\
\hline
\end{tabular}

${ }^{1}$ Median, $1{ }^{\text {st }}$ and $3{ }^{\text {rd }}$ quartiles; ${ }^{2}$ Mean $\pm S D ;{ }^{3}$ Groups within each moment, followed by the same uppercase letter, are not statistically different ( $\left.p>0.05\right) ;{ }^{4}$ Moments within each group, followed by the same lowercase letter are not statistically different ( $p>0.05) ;{ }^{*} p>0.05$ (among groups within each moment, and among moments within each group); Note: $\mathrm{A}>\mathrm{B}>\mathrm{C}$; $\mathrm{a}>\mathrm{b}>\mathrm{c}$

Table III - Systolic Index (SI), Systemic Vascular Resistance Index (SVRI), Pulmonary Vascular Resistance Index (PVRI), Left Ventricle Systolic Work Index (LVSWI), Right Ventricle Systolic Work Index (RVSWI) within Studied Moments and Groups

\begin{tabular}{|c|c|c|c|c|c|}
\hline \multirow[t]{2}{*}{ Attributes } & \multirow[t]{2}{*}{ Groups } & \multicolumn{4}{|c|}{ Moments } \\
\hline & & $M_{1}$ & $\mathrm{M}_{2}$ & $\mathrm{M}_{3}$ & $\mathrm{M}_{4}$ \\
\hline \multirow[t]{3}{*}{$\mathrm{SI}^{2 *}\left(\mathrm{ml}\right.$.beat. $\left.\mathrm{min}^{-1}\right)$} & G1 & $33.0 \pm 10.9$ & $36.6 \pm 8.9$ & $37.7 \pm 7.6$ & $35.7 \pm 5.8$ \\
\hline & G2 & $34.0 \pm 11.9$ & $37.9 \pm 9.2$ & $36.8 \pm 11.5$ & $31.9 \pm 9.9$ \\
\hline & G3 & $34.4 \pm 8.0$ & $36.4 \pm 10.0$ & $35.8 \pm 9.9$ & $29.5 \pm 7.1$ \\
\hline \multirow[t]{2}{*}{ SVRI $_{1}\left(\right.$ dina.s. $\left.\mathrm{cm}^{-5} \cdot \mathrm{m}^{-2}\right)$} & G1 & $1862[1669 ; 2460] \mathrm{A}^{3} \mathrm{a}^{4}$ & 1594 [1481; 2078] Bb & 1342 [1203; 1703] Bd & $1463[1388 ; 1967] \mathrm{Bc}$ \\
\hline & G3 & $2278[1827 ; 2457] \mathrm{Aa}$ & 2414 [2034; 2923] Aa & $2200[1855 ; 2831] \mathrm{Aa}$ & $2428[2234 ; 3088] \mathrm{Aa}$ \\
\hline \multirow[t]{3}{*}{ PVRI ${ }^{1 *}\left(\right.$ dina $\left.. s . \mathrm{cm}^{-5} \cdot \mathrm{m}^{-2}\right)$} & G1 & $154[128 ; 190]$ & $164[128 ; 191]$ & $131[110 ; 171]$ & $142[123 ; 202]$ \\
\hline & $\mathrm{G} 2$ & $166[118 ; 198]$ & $172[165 ; 192]$ & $188[121 ; 236]$ & $169[125 ; 256]$ \\
\hline & G3 & $147[118 ; 182]$ & $155[132 ; 180]$ & $159[139 ; 223]$ & $154[117 ; 174]$ \\
\hline \multirow[t]{2}{*}{ LVSWI $^{2 *}\left(\mathrm{~g} \cdot \mathrm{m} \cdot \mathrm{m}^{-2}\right)$} & G1 & $43.8 \pm 13.0$ & $43.5 \pm 13.9$ & $43.7 \pm 12.3$ & $43.8 \pm 11.9$ \\
\hline & G3 & $44.9 \pm 16.5$ & $42.7 \pm 16.3$ & $41.6 \pm 12.5$ & $37.1 \pm 10.8$ \\
\hline \multirow[t]{3}{*}{$\mathrm{RVSWI}^{2}$ * $\left(\mathrm{g} \cdot \mathrm{m} \cdot \mathrm{m}^{-2}\right)$} & G1 & $4.4 \pm 1.9$ & $4.6 \pm 2.0$ & $5.2 \pm 1.5$ & $5.1 \pm 1.6$ \\
\hline & G2 & $4.3 \pm 2.0$ & $3.7 \pm 1.5$ & $4.3 \pm 1.5$ & $3.8 \pm 1.0$ \\
\hline & G3 & $4.1 \pm 1.6$ & $3.8 \pm 1.4$ & $3.9 \pm 1.2$ & $3.2 \pm 1.4$ \\
\hline
\end{tabular}

${ }^{1}$ Median, $1{ }^{\text {st }}$ and $3{ }^{\text {rd }}$ quartiles; ${ }^{2}$ Mean $\pm S D ;{ }^{3}$ Groups within each moment, followed by the same uppercase letter, are not statistically different ( $\left.p>0.05\right) ;{ }^{4}$ Moments within each group, followed by the same lowercase letter are not statistically different ( $p>0.05) ;{ }^{*} p>0.05$ (among groups within each moment, and among moments within each group); Note: $A>B ; a>b>c$ 
Table IV - Oxygen Pulse Saturation $\left(\mathrm{SpO}_{2}\right)$, End $\mathrm{CO}_{2}$ Expiratory Pressure $\left(\mathrm{P}_{\mathrm{ET}} \mathrm{CO}_{2}\right)$, Hematocrit $(\mathrm{Ht})$ and Esophageal Temperature $(\mathrm{T})$ within Studied Moments and Groups

\begin{tabular}{|c|c|c|c|c|c|}
\hline \multirow[t]{2}{*}{ Attributes } & \multirow[t]{2}{*}{ Groups } & \multicolumn{4}{|c|}{ Moments } \\
\hline & & $M_{1}$ & $\mathrm{M}_{2}$ & $M_{3}$ & $\mathrm{M}_{4}$ \\
\hline \multirow[t]{3}{*}{$\mathrm{SpO}_{2}{ }^{1 *}(\%)$} & $\mathrm{G} 1$ & $99.0[99.0 ; 99.0]$ & $99.0[99.0 ; 99.0]$ & $98.5[98.0 ; 99.0]$ & $98.0[98.0 ; 99.0]$ \\
\hline & G2 & 99.0 [99.0; 99.0] & $99.0[99.0 ; 99.0]$ & 99.0 [98.0; 99.0] & $99.0[98.0 ; 99.0]$ \\
\hline & G3 & $99.0[98.5 ; 99.5]$ & 99.0 [99.0; 99.5] & $99.0[98.5 ; 99.0]$ & $99.0[98.0 ; 99.0]$ \\
\hline \multirow[t]{2}{*}{$\mathrm{P}_{\mathrm{ET}} \mathrm{CO}_{2}{ }^{2 *}(\mathrm{mmHg})$} & G1 & $37.2 \pm 3.8$ & $38.1 \pm 4.7$ & $39.2 \pm 3.6$ & $40.3 \pm 4.5$ \\
\hline & G3 & $36.2 \pm 5.4$ & $36.8 \pm 6.5$ & $37.2 \pm 6.7$ & $37.1 \pm 7.1$ \\
\hline \multirow[t]{3}{*}{$\mathrm{Ht}^{1 *}(\%)$} & G1 & $39.5[36.0 ; 43.0]$ & $40.0[36.5 ; 44.0]$ & $41.0[37.0 ; 43.5]$ & $41.0[37.0 ; 42.5]$ \\
\hline & $\mathrm{G} 2$ & $41.0[38.5 ; 44.5]$ & $42.0[36.0 ; 46.0]$ & $41.5[39.0 ; 46.5]$ & $43.5[40.0 ; 46.0]$ \\
\hline & G3 & $39.5[36.5 ; 42.0]$ & $41.0[35.5 ; 44.0]$ & $41.5[36.0 ; 44.0]$ & $41.5[36.0 ; 45.0]$ \\
\hline \multirow[t]{2}{*}{$\mathrm{T}^{1 *}\left({ }^{\circ} \mathrm{C}\right)$} & G1 & $38.0[37.3 ; 38.3]$ & $37.8[36.9 ; 38.7]$ & $38.1[37.5 ; 38.9]$ & $38.4[37.6 ; 39.0]$ \\
\hline & G3 & $36.9[36.4 ; 38.3]$ & $37.2[36.2 ; 38.1]$ & $37.0[36.6 ; 38.7]$ & $37.3[36.5 ; 38.7]$ \\
\hline
\end{tabular}

${ }^{1}$ Median, $1^{\text {st }}$ and $3^{\text {rd }}$ quartiles; ${ }^{2}$ Mean $\pm S D ;{ }^{*} p>0.05$ (among groups within each moment, and among moments within each group)

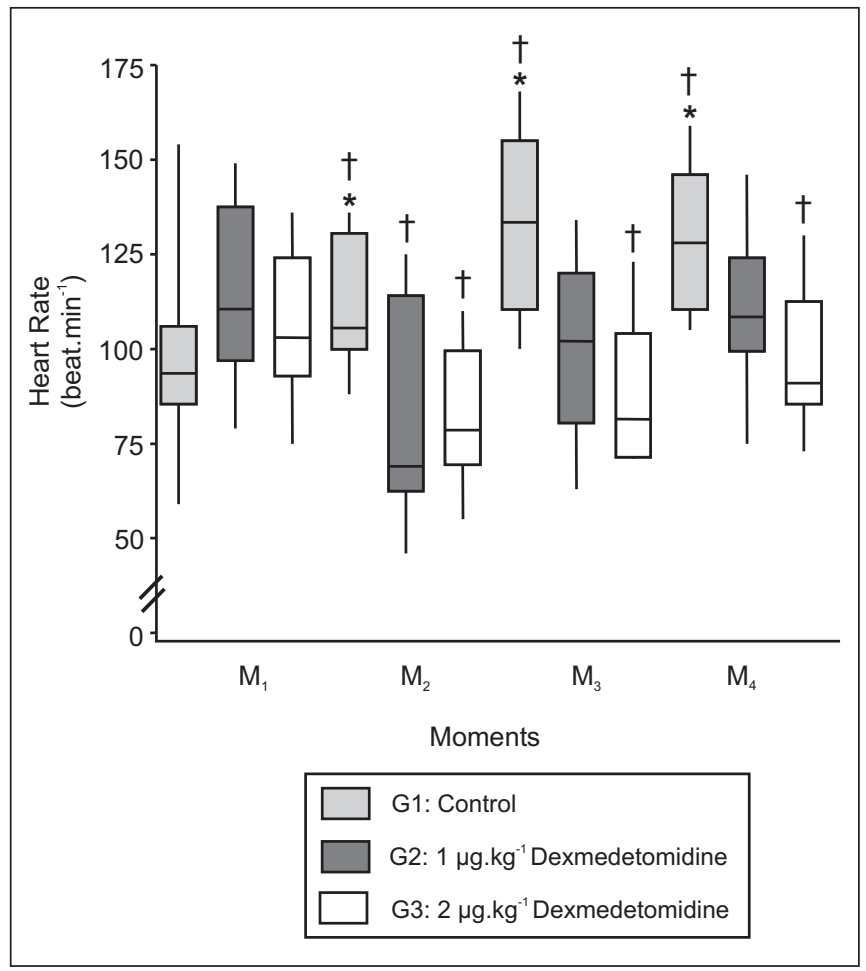

Figure 1 - Heart Rate. Median, $1^{\text {st }}$ and $3^{\text {rd }}$ Quartiles, Maximum and Minimum Values Obtained within Studied Groups and Mmoments

${ }_{*} p<0.05$ among groups within each moment $\dagger p<0.05$ among moments within each group

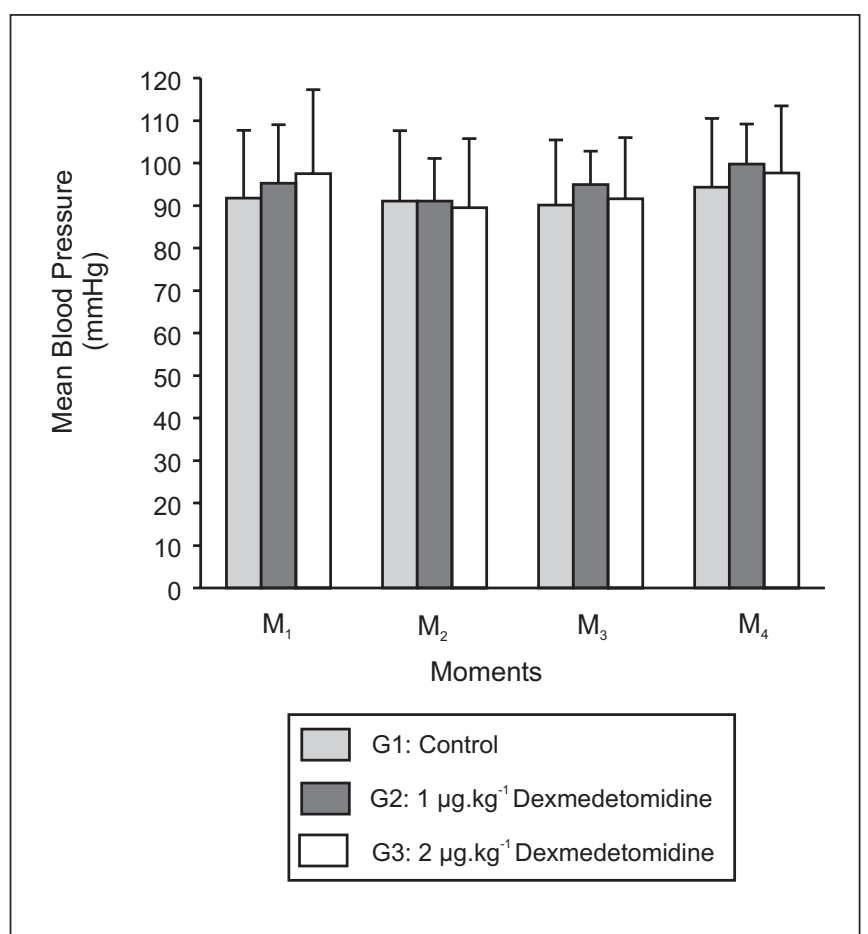

Figure 2 - Mean Blood Pressure, Mean and Standard Deviation of Values Observed within each Moment in all Studied Groups $(p>0.05)$ 


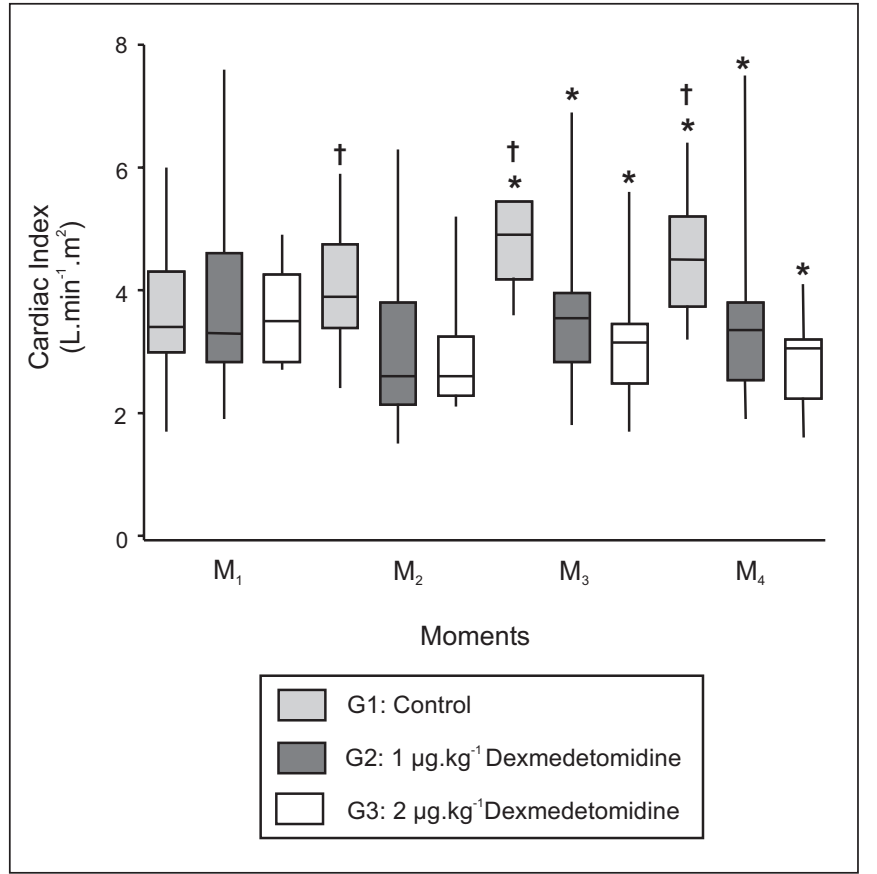

Figure 3 - Cardiac Index. Median, $1^{\text {st }}$ and $3^{\text {rd }}$ Quartiles, Maximum and Minimum Values Obtained with in Studied Groups and Moments

${ }^{*} p<0.05$ among groups within each moment

$+p<0.05$ among moments within each group

\section{DISCUSSION}

Hemodynamic variables were found within normal ranges at control moment for dogs at rest ${ }^{20}$ as a result of the good hemodynamic stability provided by the balanced anesthetic technique ${ }^{21}$ and of the adequate animals' hydration.

Similar to this experiment, several studies have reported HR decrease after dexmedetomidine injection ${ }^{8,22-24}$. Bloor et al. ${ }^{25}$ have described HR decrease after $20 \mu \mathrm{g} . \mathrm{kg}^{-1}$ dexmedetomidine injection in dogs in 2-minute interval, preceded of MBP increase. Dyck et al. ${ }^{26}$ have also observed MBP increase and HR decrease after $2 \mu \mathrm{g} . \mathrm{kg}^{-1}$ intravenous dexmedetomidine in volunteers, in 5-minute interval; these changes however have not been observed with intramuscular injections. These authors have suggested that one mechanism involved in HR decrease after dexmedetomidine injection is baroreflex activation and slow drug injection would tend to minimize it. Our study has shown HR decrease with no previous MBP increase. Several other authors have also observed HR decrease without MBP changes after dexmedetomidine injection ${ }^{27-29}$. In addition to baroreflex activation, other factors may be involved in HR decrease after dexmedetomidine injection, such as parasympathetic nervous system activation increase by solitaire tract nucleus stimulation ${ }^{30,31}$, central sympathetic nervous system (SNS) activity decrease ${ }^{32}$ or catecholamine release inhibition in SNS nervous terminations ${ }^{7,27,33}$. We have observed that HR decrease was higher and lasted longer with the highest dexmedetomidine dose, confirming that this change is

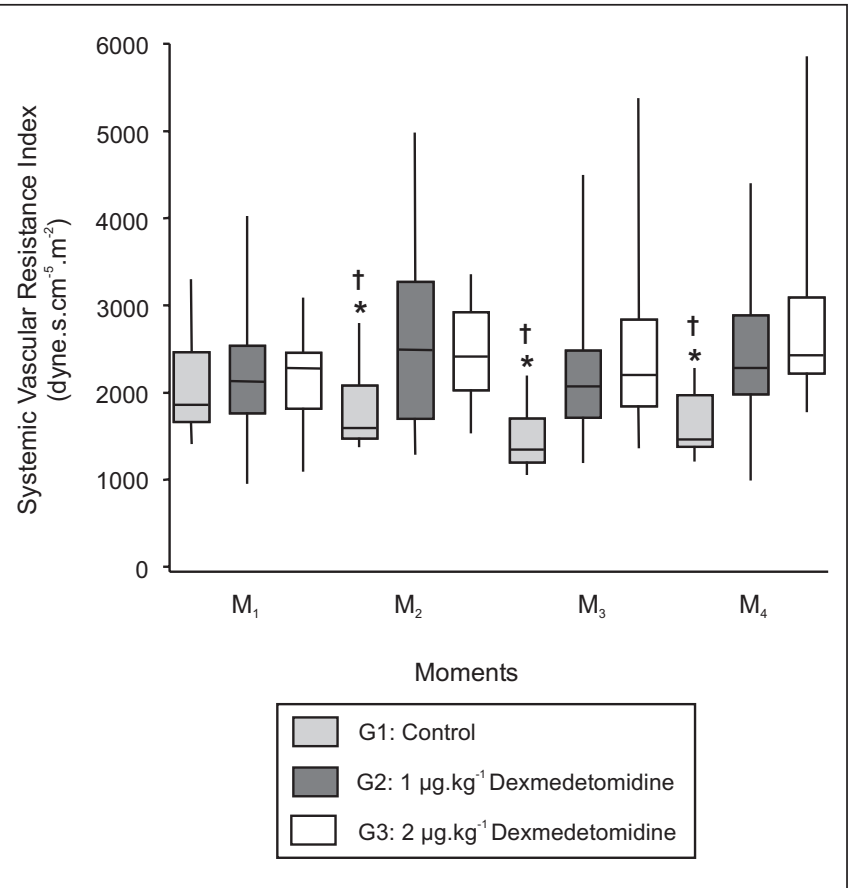

Figure 4 - Systemic Vascular Resistance Index. Median, $1^{\text {st }}$ and $3^{\text {rd }}$ Quartiles, Maximum and Minimum Values Obtained within Studied Groups and Moments

${ }^{*} p<0.05$ among groups within each moment

$+p<0.05$ among moments within each group

dose-dependent ${ }^{34}$. HR was increased throughout the experiment in the control group, probably due to isoflurane-induced baroreflex activation, or to SNS hyperactivity due to anesthetic maintenance with 0.6 MAC isoflurane, what was not observed in groups receiving dexmedetomidine, since this drug decreases isoflurane MAC in up to $70 \%{ }^{6}$.

Alpha $_{2}$-adrenergic receptors are classified according to their pharmacological action in $\alpha_{2} A, \alpha_{2} B$ and $\alpha_{2} \mathrm{C}$. Pre-junctional $\alpha_{2}$ receptors are primarily $\alpha_{2} A$, although they may also be present in $\alpha_{2} \mathrm{C}$ form ${ }^{35}$. They inhibit norepinephrine release in sympathetic nervous terminations and in central nervous system noradrenergic neurons ${ }^{36}$. All three receptor subtypes may be identified in vessels smooth muscles and promote vasoconstriction, being $\alpha_{2} \mathrm{C}$ primarily located in veins ${ }^{36}$. Dexmedetomidine is also bound to imidazolic receptors and it is possible that some hemodynamic changes observed after the injection of such drug are also mediated by those receptors $^{37}$

Several authors have observed that dexmedetomidine promotes a two-phase MBP change, with initial increase and further decrease ${ }^{8,26}$. Ebert et al. ${ }^{9}$ have reported that dexmedetomidine concentrations below $1.2 \mathrm{ng} . \mathrm{ml}^{-1}$ induce MBP decrease, while concentrations above $1.9 \mathrm{ng} \cdot \mathrm{ml}^{-1}$ would trigger a progressive MBP increase due to the prevalence of the drug action on post-junctional $\alpha_{2}$ receptors. In our study, there has been no significant MBP change after dexmedetomidine injection. Several authors have reported that dexmedetomidine injection in dogs would primarily promote MBP increase ${ }^{24,38,39}$. This, however, was not reproVol. 53, № 6, Novembro - Dezembro, 2003 
duced in our study, probably because, as opposed to other authors, we have used lower dexmedetomidine doses, similar to those used in the clinical practice, or even by isoflurane's action blocking dexmedetomidine's vasoconstriction $^{40}$.

Although dexmedetomidine decreases MBP acting on pre-junctional $\alpha_{2}$ receptors, paradoxically there is systemic vascular resistance increase after high dexmedetomidine doses $^{23}$. Differently from other authors ${ }^{23-25}$, we have not observed SVRI increase in dogs receiving dexmedetomidine. Two factors may have accounted for this difference. First, dexmedetomidine dose used in this study was lower than those used in previous experiments and, second, isoflurane, for being a vasodilating agent ${ }^{41}$, has counterbalanced dexmedetomidine vasoconstrictor response. So, although not observing SVRI increase with dexmedetomidine, this drug was able to prevent SVRI decrease observed in the control group, but not in treated groups.

In previous studies, dexmedetomidine was able to dose-dependently decrease $\mathrm{Cl}$ by decreasing HR, increasing afterload $^{9,24}$ or impairing myocardial contractility by coronary flow reduction ${ }^{23}$. Dexmedetomidine doses used in this experiment have not significantly changed $\mathrm{Cl}$, but have prevented progressive and significant $\mathrm{Cl}$ increase observed in the control group, for decreasing $\mathrm{HR}$, preventing isoflurane-induced afterload decrease and inhibiting autonomic response triggered by superficial anesthetic level in $\mathrm{G} 1$.

PAP has increased in $\mathrm{G} 1$, but not in groups receiving dexmedetomidine. This PAP increase was the result of HR increase observed in the control group.

In our study, dexmedetomidine has not promoted PCWP, RAP, SI, PVRI, RVSWI and LVSWI changes, confirming previous studies results ${ }^{10,15,34}$ which have shown that with low dexmedetomidine doses, close to those used in the clinical practice, there is sedation, analgesia, norepinephrine plasma concentration and HR decrease, without however significantly changing hemodynamic parameters.

We concluded that dexmedetomidine in dogs, in the conditions of our study, has promoted dose-dependent HR decrease, has not changed MBP, has prevented Cl increase observed in the control group, and has inhibited isoflurane-induced SVRI decrease, proving to be safe and able to promote satisfactory hemodynamic stability.

\section{REFERÊNCIAS - REFERENCES}

01. Udelsman R, Norton JA, Jelenich SE et al - Responses of the hypothalamic-pituitary-adrenal and renin-angiotensin axes and the sympathetic system during controlled surgical and anesthetic stress. J Clin Endocrinol, 1987;64:986-994.

02. Oliver MF, Goldman L, Julian DG et al - Effects of mivazerol on perioperative cardiac complications during non-cardiac surgery in patients with coronary heart disease: the European mivazerol trial (RMIT). Anesthesiology, 1999;91:951-961.
03. Hayashi $Y$, Maze M - Alpha 2 adrenoceptor agonists and anaesthesia. Br J Anaesth, 1993;71:108-118.

04. Karol MD, Maze M - Pharmacokinetics and interaction pharmacodynamics of dexmedetomidine in humans. Baillière's Clin Anesthesiol, 2000;14:261-269.

05. Doze VA, Chen BX, Maze M - Dexmedetomidine produces a hypnotic-anesthetic action an rats via activation of $\alpha_{2}$-adrenoceptors. Anesthesiology, 1989;71:75-79.

06. Nguyen D, Abdul-Rasool I, Ward D et al - Ventilatory effects of dexmedetomidine, atipamezole, and isoflurane in dogs. Anesthesiology, 1992;76:573-579.

07. Scheinin M, Kallio A, Koulu M et al - Sedative and cardiovascular effects of medetomidine, a novel selective alpha ${ }_{2}$ adrenoceptor agonist, in healthy volunteers. Br J Clin Pharmacol, 1987;4: 443-451.

08. Kallio A, Scheinin M, Koulu M et al - Effects of dexmedetomidine, a selective $\alpha_{2}$-adrenoceptor agonist, on hemodynamic control mechanisms. Clin Pharmacol Ther, 1989;46:33-42.

09. Ebert TJ, Hall JE, Barney JA et al - The effects of increasing plasma concentrations of dexmedetomidine in humans. Anesthesiology, 2000;93:382-394.

10. Aho M, Scheinin M, Lehtinen AM et al - Intramuscularly administered dexmedetomidine attenuates hemodynamic and stress hormone responses to gynecologic laparoscopy. Anesth Analg, 1992;75:932-939.

11. Fragen RJ, Fitzgerald PC - Effect of dexmedetomidine on the minimum alveolar concentration (MAC) of sevoflurane in adult age 55 to 70 years. J Clin Anesth, 1999;11:466-470.

12. Nociti JR, Serzedo PSM, Zuccolotto EB et al - Dexmedetomidina associada a propofol em sedação durante anestesia local para cirurgia plástica. Rev Bras Anestesiol 2003;53:198-208.

13. Aho M, Lehtnen AM, Erkola O et al - The effect of intravenously administered dexmedetomidine on perioperative hemodynamics and isoflurane requirements in patients undergoing abdominal hysterectomy. Anesthesiology, 1991,74: 997-1002.

14. Curtis FG, Castiglia YMM, Stolf AA et al - Dexmedetomidina e sufentanil como analgésicos per-operatórios. Estudo comparativo. Rev Bras Anestesiol 2002;52:525-534.

15. Talke P, Chen R, Thomas B et al - The hemodynamic and adrenergic effects of perioperative dexmedetomidine infusion after vascular surgery. Anesth Analg, 2000;90:834-839.

16. Venn RM, Bradshaw CJ, Spencer R et al - Preliminary UK experience of dexmedetomidine, a novel agent for postoperative sedation in the intensive care unit. Anaesthesia, 1999;54: 1136-1142.

17. Aantaa R, Kallio A, Virtanen R - Dexmedetomidine, a novel $\alpha_{2}$-adrenergic agonist. A review of its pharmacodynamic characteristics. Drugs Future, 1993;18:49-56.

18. Villela NR, Nascimento Jr P - Uso de dexmedetomidina em Anestesiologia. Rev Bras Anestesiol, 2003;53:97-113.

19. Jones MEP, Maze M - Can we characterize the central nervous system action of $\alpha_{2}$-adrenergic agonists? $\mathrm{Br} \mathrm{J}$ Anaesth, 2001;86:1-3.

20. Massone F - Anestesiologia Veterinária. $3^{\mathrm{a}}$ Ed, Rio de Janeiro: Guanabara Koogan, 1999.

21. Monk TG, Mueller M, White PF - Treatment of stress response during balanced anesthesia. Comparative effects of isoflurane, alfentanil and trimethaphan. Anesthesiology, 1992;76:39-45.

22. Vickery RG, Sheridon BC, Segal IS et al - Anesthetic and hemodynamic effects of the steroisomers of medetomidine, an alpha2-adrenergic agonist, in halothane-anesthetized dogs. Anesth Analg, 1988;67:611-615. 
23. Flacke JW, Flacke WE, Bloor BC et al - Hemodynamic effects of dexmedetomidine, an $\alpha_{2}$-adrenergic agonist, in autonomically denervated dogs. J Cardiovasc Pharmacol, 1990;16:616-623.

24. Schmeling WT, Kampine JP, Roerig DL et al - The effects of stereoisomers of the $\alpha_{2}$-adrenergic agonist medetomidine on systemic and coronary hemodynamics in conscious dogs. Anesthesiology, 1991;75:499-511.

25. Bloor BC, Alper G, Frankland M et al - Nifedipine attenuates the acute hemodynamic changes that occur with intravenous dexmedetomidine in dogs. Anesth Analg, 1989;68:S32.

26. Dyck JB, Maze M, Haack $C$ et al - The pharmacokinetics and hemodynamic effects of intravenous and intramuscular dexmedetomidine hydrochloride in adult human volunteers. Anesthesiology, 1993;78:813-820.

27. Proctor LT, Schmeling WT, Roerig D et al - Oral dexmedetomidine attenuates hemodynamic responses during emergence from general anesthesia in chronically instrumented dogs. Anesthesiology, 1991;74:108-114.

28. Zornow MH - Ventilatory, hemodynamic and sedative effects of the $\alpha_{2}$-adrenergic agonist, dexmedetomidine. Neuropharmacology, 1991;30:1065-1071.

29. Oku S, Benson KT, Hirakawa M et al - Renal sympathetic nerve activity after dexmedetomidine in nerve-intact and baroreceptor-denervated rabbits. Anesth Analg, 1996;83: 477-481.

30. Mroczek WJ, Davidov M, Finnerty FA - Intravenous clonidine in hypertensive patients. Clin Pharmacol Ther, 1973;14:847-851.

31. Reis DJ, Morrison S, Ruggiero DA-The C1 area of the brainstem in tonic and reflex control of the circulation. Hypertension, 1988;11:8-13

32. Svennson TH, Bunney BS, Aghajanian GK - Inhibition of both noradrenergic and serotonergic neurons in brain by the alpha-adrenergic agonist clonidine. Brain Res, 1975;92:291-306.

33. Engelhard K, Werner C, Kaspar S et al - Effects of the $\alpha_{2}$-agonist dexmedetomidine on cerebral neurotransmitter concentrations during cerebral ischemia in rats. Anesthesiology 2002;96: 450-457.

34. Ebert TJ, Hall JE, Barney JA et al - The effects of increasing plasma concentrations of dexmedetomidine in humans. Anesthesiology, 2000;93:382-394.

35. Docherty JR - Subtypes of functional $\alpha_{1}$ - and $\alpha_{2}$-adrenoceptors. Eur J Pharmacol, 1998;361:1-15.

36. Saunders C, Limbird LE - Localization and trafficking of $\alpha_{2}$-adrenergic receptor subtypes in cells and tissues. Pharmacol Ther, 1999;84:193-205.

37. Khan ZP, Furgson CN, Jones RM - Alpha-2 and imidazoline receptor agonists. Their pharmacology and therapeutic role. Anesthesia, 1999;54:146-165.

38. Kuusela E, Raekallio M, Anttila M et al - Clinical effects and pharmacokinetics of medetomidine and enantiomers in dogs. $J$ Vet Pharmacol Therap, 2000;23:15-20.

39. Lawrence CJ, Prinzen FW, de Lange S - Comparison of the haemodynamic effects of the alpha-2 agonists clonidine and dexmedetomidine in the anaesthetized dog. $\mathrm{J}$ Cardiothorac Vasc Anesth, 1994;8:32.
40. Larach DR, Schuler HG, Derr JA et al - Halothane selectively attenuates alpha $_{2}$-adrenoceptor mediated vasoconstriction, in vivo and in vitro. Anesthesiology, 1987;66:781-792.

41. Stevens WC, Cromwell TH, Hasey MJ et al - The cardiovascular effects of a new inhalation anesthetic, forane, in human volunteers at constant arterial carbon dioxide tension. Anesthesiology, 1971;35:8-16

\section{RESUMEN}

Villela NB, Nascimento Jr P, Carvalho LR - Efectos Cardiovasculares de Dos Dosis de Dexmedetomidina. Estudio Experimental en Canes

JUSTIFICATIVA Y OBJETIVOS: La dexmedetomidina es un nuevo agonista $\alpha_{2}$-adrenérgico, $y$ en la actualidad hay un creciente interés en su uso en Anestesiología, por reducir el consumo de anestésicos y promover estabilidad hemodinámica. El objetivo de esta pesquisa fue estudiar los efectos cardiovasculares de la dexmedetomidina en el can anestesiado, empleándose dos dosis distintas y semejantes a aquellas utilizadas en Anestesiología.

MÉTODO: 36 canes adultos anestesiados con propofol, fentanil e isoflurano fueron divididos en tres grupos: $G 1$, inyección de $20 \mathrm{ml}$ de solución de clorato de sodio a 0,9\%, en 10 minutos, seguida de inyección de $20 \mathrm{ml}$ de la misma solución, en 1 hora; G2, inyección de $20 \mathrm{ml}$ de solución de clorato de sodio a $0,9 \%$ conteniendo dexmedetomidina (1 $\mu \mathrm{g} . \mathrm{kg}^{-1}$ ), en 10 minutos, seguida de inyección de $20 \mathrm{ml}$ de la misma solución, en 1 hora y G3, inyección de 20 ml de solución de clorato de sodio a 0,9\% conteniendo dexmedetomidina (2 $\mu \mathrm{g} . \mathrm{kg}^{-1}$ ) en 10 minutos, seguida de inyección de $20 \mathrm{ml}$ de la misma solución, en 1 hora. Se estudiaron los atributos cardiovasculares en cuatro momentos: $M_{1}$, control; $M_{2}$, después de la inyección inicial de $20 \mathrm{ml}$ de la solución en estudio, en 10 minutos, coincidiendo con el inicio de la inyección de la misma solución, en 1 hora; $M_{3}, 60$ minutos después $M_{2}$ y $M_{4}, 60$ minutos después $M_{3}$.

RESULTADOS: La frecuencia cardíaca (FC) diminuyó en el $G 2$, en el $M_{2}$, retornando a los valores basales en el $M_{3}$, en cuanto en el G3 diminuyó en el $M_{2}$, manteniéndose baja durante todo el experimento. En el $\mathrm{G} 1$ hubo aumento progresivo de la FC. En ningún grupo hubo alteración de la presión arterial. La resistencia vascular sistémica (RVS) se mantuvo estable en el G2 y G3, en cuanto en el G1 presentó reducción en $M_{2}$, manteniéndose baja durante el experimento. El índice cardíaco (IC) no presentó alteraciones significativas en el G2 y G3, más aumentó progresivamente en el G1.

CONCLUSIONES: Se concluye que en el can, en las condiciones experimentales empleadas, la dexmedetomidina diminuye la FC de forma dosis dependiente, inhibe la reducción de la RVS producida por el isoflurano e impide la ocurrencia de respuesta hiperdinámica durante el experimento. 